\title{
Distributed Hybrid Scheduling in Multi-Cloud Networks using Conflict Graphs
}

\author{
Ahmed Douik, Student Member, IEEE, Hayssam Dahrouj, Senior Member, IEEE, \\ Tareq Y. Al-Naffouri, Member, IEEE, and Mohamed-Slim Alouini, Fellow, IEEE
}

\begin{abstract}
Recent studies on cloud-radio access networks assume either signal-level or scheduling-level coordination. This paper considers a hybrid coordinated scheme as a means to benefit from both policies. Consider the downlink of a multicloud radio access network, where each cloud is connected to several base-stations (BSs) via high capacity links, and, therefore, allows for joint signal processing within the cloud transmission. Across the multiple clouds, however, only scheduling-level coordination is permitted, as low levels of backhaul communication are feasible. The frame structure of every BS is composed of various time/frequency blocks, called power-zones (PZs), which are maintained at a fixed power level. The paper addresses the problem of maximizing a network-wide utility by associating users to clouds and scheduling them to the PZs, under the practical constraints that each user is scheduled to a single cloud at most, but possibly to many BSs within the cloud, and can be served by one or more distinct PZs within the BSs' frame. The paper solves the problem using graph theory techniques by constructing the conflict graph. The considered scheduling problem is, then, shown to be equivalent to a maximum-weight independent set problem in the constructed graph, which can be solved using efficient techniques. The paper then proposes solving the problem using both optimal and heuristic algorithms that can be implemented in a distributed fashion across the network. The proposed distributed algorithms rely on the well-chosen structure of the constructed conflict graph utilized to solve the maximumweight independent set problem. Simulation results suggest that the proposed optimal and heuristic hybrid scheduling strategies provide appreciable gain as compared to the scheduling-level coordinated networks, with a negligible degradation to signallevel coordination.
\end{abstract}

Index Terms-Multi-cloud networks, coordinated scheduling, scheduling-level coordination, signal-level coordination, centralized and distributed scheduling.

\section{INTRODUCTION}

Next generation mobile radio systems (5G) are expected to undergo major architectural changes, so as to support the deluge in demand for mobile data services by increasing capacity, energy efficiency and latency reduction [2], [3]. One way to boost throughput and coverage in dense data networks is by moving from the single high-powered base-station (BS)

A part of this paper [1] is published in IEEE Global Telecommunications Conference (GLOBECOM' 2015), San Diego, CA, USA.

Hayssam Dahrouj would like to thank Effat University in Jeddah, Saudi Arabia, for funding the research reported in this paper through the Research and Consultancy Institute.

Ahmed Douik is with the Department of Electrical Engineering, California Institute of Technology, Pasadena, CA 91125 USA (e-mail: ahmed.douik@caltech.edu).

Hayssam Dahrouj is with the Department of Electrical Engineering, Effat University, Jeddah 22332, Saudi Arabia (e-mail: hayssam.dahrouj@gmail.com).

T. Y. Al-Naffouri and M.-S. Alouini are with the Division of Computer, Electrical and Mathematical Sciences, and Engineering, King Abdullah University of Science and Technology, Thuwal 23955-6900, Saudi Arabia (e-mail: \{tareq.alnaffouri,slim.alouini\}@kaust.edu.sa). to the massive deployment of overlaying BSs of different sizes. Such architecture, however, is subject to high inter-BS interference, especially with the progressive move towards full spectrum reuse in $5 \mathrm{G}$. Traditionally, interference mitigation is performed by coordinating the different BSs through massive signaling and message exchange. Such coordination technique, however, in addition to being energy-inefficient [4], may not always be feasible given the capacity limits of the backhaul links.

A promising network architecture for fulfilling the ambitious metrics of $5 \mathrm{G}$ is the cloud-radio access network (CRAN) [5], [6], which is obtained by connecting the different BSs to a central unit, known as the cloud. Such architecture moves most of the fundamental network functionalities to the cloud side, thereby allowing a separation between the control plane and the data plane. The virtualization in CRANs provides efficient resource utilization, joint BSs operation (joint transmission, encoding and decoding), and efficient energy control.

Different levels of coordination in CRANs are studied in the past literature, namely the signal-level coordination [7][9], and the scheduling-level coordination [10]-[12]. In signallevel coordinated CRANs [7]-[9], all the data streams of different users are shared among the different BSs, thereby allowing joint operation. However, such level of coordination necessitates high-capacity backhaul links. On the other hand, in scheduling-level coordinated CRANs [10]-[12], the cloud is responsible only for the efficient allocation of the resource blocks of each BS, which requires much less backhauling. While more practical to implement, scheduling-level coordination may lead to an inferior performance as compared to signal-level coordination. While clouds are typically connected to their base-stations through high-capacity links, cloud-tocloud communication is done via wireless links. This paper, therefore, proposes a hybrid scheduling scheme which benefits from the advantages of both scheduling policies. In particular, the paper proposes using signal-level coordination within each cloud, and scheduling-level coordination among different clouds.

Consider the downlink of a multi-CRAN, where each cloud is connected to several BSs. The frame structure of every BS is composed of various time/frequency blocks, called powerzones (PZs), kept at a fixed power level. This paper proposes a hybrid level of coordination for the scheduling problem. For BSs connected to the same cloud, associating users to PZs is performed assuming signal-level coordination. Across the multiple clouds, only scheduling-level coordination is permitted, as it requires a lower level of backhaul communication.

In this paper context, hybrid-level coordination refers to the scheme wherein multiple clouds coordinate their transmission on a scheduling-level basis only. Every cloud, however, is 
responsible for coordinating the transmission of its connected base-stations on a signal-level basis. The hybrid scheduling problem then denotes the strategy of assigning users to clouds across the network, under the system limitation that each user is scheduled at most to a single cloud since, otherwise, intercloud signal-level coordination is required. However, across the BSs connected to one cloud, users can be served by multiple BSs and different PZs within each transmit frame. Each PZ is further constrained to serve exactly one user.

\section{A. Related Work}

The paper is related in part to the classical works on scheduling, and in part to the recent works on CRAN. In the classical literature of cellular systems, scheduling is often performed assuming a prior assignment of users to BSs, e.g., the classical proportional fairness scheduling investigated in [10], [13]. In CRANs, recent works on coordinated scheduling consider a single cloud processing, as in [11], [12]. Reference [11] considers the particular case of coordinated scheduling when the number of users is equal to the number of available power-zones. Reference [11] shows that, in a context of a soft-frequency reuse, the problem reduces to a classical linear programming problem that can be solved using the auction methodology [14]. The problem is extended to an arbitrary number of users and power-zones in [12] and is shown to be an NP-hard problem. This paper is further related to the multicloud network studied in [7], [15] which, however, assume a pre-known user-to-cloud association.

Interference mitigation in CRANs via signal-level coordination has also been pivotal in the past few years. The authors in [8] consider the problem of maximizing the weighted sum-rate under finite-capacity backhaul and transmit power constraints. Unlike previous studies in which compression is performed independently of the base-station operations, the authors in [8] consider a joint precoding and backhaul compression strategy. Similarly, a clustered base transceiver station coordination strategy with clustered linear precoding is proposed in [16]. Reference [9] considers the problem of minimizing the total power consumption by accounting for the transport link power in a green-CRAN and proposes solving the problems using techniques from compressive sensing and optimization theory. Reference [17] derives bounds on the achievable ergodic capacity to quantify the user diversity gain. In a classic multicell network setup, reference [18] investigates the problem of joint beamforming design in a multi-cell system where multiple base-stations can serve each scheduled user. Using compressive sensing technique, reference [18] illustrates the interplay between the transmit sum-power and the backhaul sum-capacity required to form the clusters, under fixed signalto-interference-and-noise ratio (SINR) constraints.

All the aforementioned network optimization algorithms are centralized in nature, which is not always practically feasible for computational complexity reasons. This paper addresses this issue by proposing distributed algorithms so as to lessen the computational complexity and facilitate the practical implementation of the proposed methods. The paper is, therefore, related to the recent state-of-art on distributed scheduling, e.g., [19]-[24]. While reference [19] considers maximizing the capacity based on the complete co-channel gain information, reference [20] considers the average channel state information only. Reference [21], on the other hand, proposes a distributed algorithm for interference mitigation which automatically adjusts the transmit power in orthogonal frequency division multiple access (OFDMA) based cellular systems. Reference [22], further, investigates a distributed scheduling approach to maximize the sum-rate using zeroforcing beamforming in a multiple-antenna base-station setup. Reference [22], particularly, shows that distributing the computations of the scheduling problem across the users provides satisfactory results by decreasing the computational complexity and reducing overhead. A survey on useful distributed techniques can be found in [24].

The distributed algorithms presented in this paper are also related to solutions suggested in [23], [25]-[28]. References [23], [25]-[28], however, are based on a game-theoretical formulation of the resource allocation problem. For instance, reference [25] proposes a distributed algorithm for resource allocation and adaptive transmission in a multi-cell scenario, which enables a trade-off between the aggressive reuse of the spectrum and the consequent co-channel interference. Similarly, in [28], the balance between the power and resource allocation is investigated. Finally, while reference [26] considers the distributed power control scheme in wireless ad hoc networks, reference [27] studies the problem in wireless OFDM systems.

\section{B. Contributions}

Unlike the aforementioned references, this papers considers the downlink of a multi-CRAN, where each cloud is connected to several base-stations (BSs) via high capacity links and, therefore, allows for joint signal processing within the cloud transmission. Across the multiple clouds, however, only scheduling-level coordination is permitted. The frame structure of every BS is composed of various power-zones, which are maintained at a fixed power level. The paper then addresses the coordinated scheduling with an objective of maximizing a generic utility function. The paper's main contribution is to solve the problem optimally using techniques inherited from graph theory. The paper proposes both optimal and heuristic distributed solutions to the problem. The paper also explicitly characterizes the extremes in scheduling policies, i.e., either scheduling-level or signal-level coordination, and proposes solving the problems using graph-theory based algorithms. The paper solely focuses on finding the optimal schedule both in centralized and distributed fashions. The optimization of transmit power and beamforming strategies is out of the scope of this manuscript, and left for future investigation.

The first part of the paper investigates the centralized coordinated scheduling problem. It considers the architecture wherein all the clouds are connected to a central processor that is responsible for computing the scheduling policy and maintaining the synchronization of the different transmit frames. The paper proposes solving the hybrid scheduling problem by constructing the conflict graph, in which each 
vertex represents an association of cloud, user, base-station and power-zone. The solution then relies on reformulating the problem as a maximum-weight independent set problem that can be optimally solved using efficient algorithms, e.g., [29][32].

The second part of the paper investigates the distributed coordinated scheduling problem. It considers the scenario wherein the different clouds are connected through low capacity links. In this configuration, the optimal scheduling decision is reached through intelligent, reasonable information exchange among the clouds. The distributed solution is achieved via the construction of local conflict graphs and the local solutions of the maximum-weight independent set problem. In order to produce a feasible solution, a conflict resolution phase comes afterward to ensure that each user is scheduled to at most a single cloud. Further, the paper proposes a low complexity, heuristic, distributed solution that relies on strictly assigning users to clouds according to the highest utility.

Finally, the paper considers both the scheduling-level and signal-level coordination separately and shows how each setup can be solved as a particular case of the generic framework. The paper simulation results suggest that the proposed hybrid scheduling strategy provides appreciable gain as compared to the scheduling-level coordinated networks, with a negligible degradation to signal-level coordination.

The rest of this paper is organized as follows: In Section II, the system model, and the problem formulation are presented. Section III proposes a solution to the hybrid scheduling problem. In Section IV, optimal and heuristic distributed solutions are presented. Section V presents the scheduling solution of signal and scheduling level coordinated networks. Simulation results are discussed in Section VI, and conclusions are presented in Section VII.

\section{System Model and Problem Formulation}

\section{A. System Model and Parameters}

Consider the downlink of a multi-CRAN of $C$ clouds serving $U$ users in total. The $C$ clouds are connected to a central cloud. Each cloud (except the central one) is connected to $B$ BSs and is responsible for the signal-level coordination of the connected BSs. Figure 1 illustrates a multi-CRAN formed by $U=21$ users, and $C=3$ clouds each coordinating $B=3$ BSs. Let $\mathcal{C}$ be the set of clouds in the system each coordinating the set of BSs $\mathcal{B}$. All BSs and users are equipped with single antennas. Extension to the scenarios in which each base-station (respectively user) have multiple antenna can be obtained by considering each antenna as a separate basestation (respectively user), i.e., no beamforming is allowed across the multiple antennas. Beamforming optimization is left for future investigation. Let $\mathcal{U}$ be the set of users in the network with $|\mathcal{U}|=U$, where the notation $|\mathcal{X}|$ refers to the cardinality of a set $\mathcal{X}$. The transmit frame of each BS is composed of several time/frequency resource blocks maintained at fixed transmit power. In this paper, the generic term PZ is used to refer to a time/frequency resource block of a BS. Let $\mathcal{Z}$ be the set of the $Z \mathrm{PZs}$ of the frame of one BS. This paper assumes

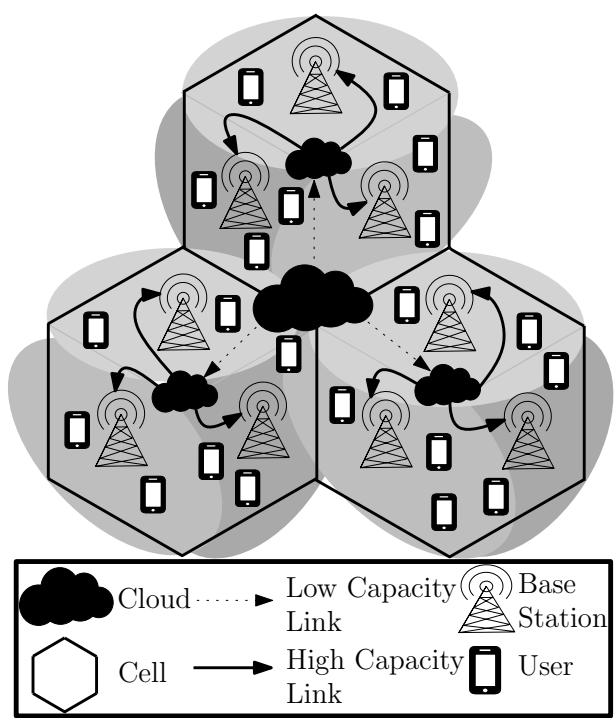

Fig. 1. Cloud enabled network composed 3 cells, each containing 3 base stations and 7 users.

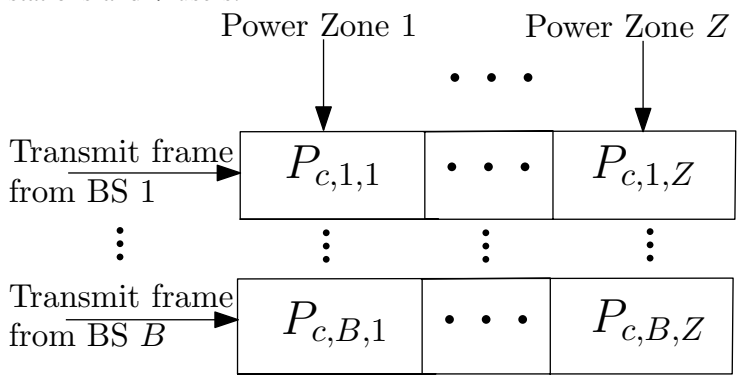

Fig. 2. Frame structure of $B$ base stations each containing $Z$ power zones.

that the number of base-stations within each cloud and the number of power-zones in each base-station is the same to simplify the notation. However, all the results and algorithms presented in this manuscript are agnostic to the aforementioned assumption. The transmit power of the $z$ th $\mathrm{PZ}$ in the $b$ th $\mathrm{BS}$ of the $c$ th cloud is fixed to $P_{c b z}, \forall(c, b, z) \in \mathcal{C} \times \mathcal{B} \times \mathcal{Z}$, where the notation $\mathcal{X} \times \mathcal{Y}$ refers to the Cartesian product of the two sets $\mathcal{X}$ and $\mathcal{Y}$. Figure 2 shows the coordinated frames of the connected BSs in the $c$ th cloud. Note that this paper focuses on the scheduling optimization, i.e., for a fixed transmit paper. Optimization with respect to the power values $P_{c b z}$ is left for future research, e.g., using the framework of [33].

Each cloud $c \in \mathcal{C}$ is responsible for coordinating its $B$ BSs, which allows joint signal processing across them. The central cloud connecting all the clouds $c \in \mathcal{C}$ is responsible for computing the scheduling policy, and also guarantees that the transmission of the different frames are synchronized across all BSs in the network ( $C B$ BSs). Let $h_{c b z}^{u} \in \mathbb{C}, \forall(c, u, b, z) \in$ $\mathcal{C} \times \mathcal{U} \times \mathcal{B} \times \mathcal{Z}$ be the complex channel gain from the $b$ th BS of the $c$ th cloud to user $u$ scheduled to $\mathrm{PZ} z$. The signal-tointerference plus noise-ratio (SINR) of user $u$ when scheduled to $\mathrm{PZ} z$ in the $b$ th $\mathrm{BS}$ of the $c$ th cloud can be expressed as:

$$
\operatorname{SINR}_{c b z}^{u}=\frac{P_{c b z}\left|h_{c b z}^{u}\right|^{2}}{\Gamma\left(\sigma^{2}+\sum_{\left(c^{\prime}, b^{\prime}\right) \neq(c, b)} P_{c^{\prime} b^{\prime} z}\left|h_{c^{\prime} b^{\prime} z}^{u}\right|^{2}\right)},
$$

where $\Gamma$ denotes the SINR gap, i.e., the discrepancy between the maximum information theoretical achievable capacity and 
the one achieved by the used channel code, constellation, and channel distribution. The Gaussian noise variance is denoted by $\sigma^{2}$. This paper assumes that the cloud is able to perfectly estimate all the values of the channel gains $h_{c b z}^{u}$ and thus the different SINRs.

\section{B. Scheduling Problem Formulation}

The scheduling problem under investigation in this paper consists of assigning users to clouds and scheduling them to PZs in each BS frame under the following practical constraints.

- C1: Each user can connect at most to one cloud but possibly to many BSs in that cloud.

- C2: Each PZ should be allocated to exactly one user.

- C3: Each user cannot be served by the same PZ across different BSs.

where the first constraint translates the fact that only scheduling-level coordination is allowed between the different clouds, condition $\mathrm{C} 2$ specifies that all radio resources need to be utilized for best performance, and $\mathrm{C} 3$ forbids beamforming across the connected base-stations.

Let $\pi_{c u b z}$ be a generic network-wide benefit of assigning user $u$ to the $z$ th PZ of the $b$ th BS in the $c$ th cloud. Let $X_{c u b z}$ be a binary variable that is 1 if user $u$ is mapped to the $z$ th PZ of the $b$ th BS in the $c$ th cloud, and zero otherwise. Similarly, let $Y_{u z}$ be a binary variable that is 1 if user $u$ is mapped to the $z$ th PZ of any BS across the network, and zero otherwise. Further, let $Z_{c u}$ be a binary variable that is 1 if user $u$ is assigned to cloud $c$. The scheduling problem this paper addresses can be formulated as the following 0-1 mixed integer programming problem:

$$
\begin{aligned}
\max & \sum_{c, u, b, z} \pi_{c u b z} X_{c u b z} \\
\text { s.t. } & Z_{c u}=1-\delta\left(\sum_{b, z} X_{c u b z}\right), \forall(c, u) \in \mathcal{C} \times \mathcal{U}, \\
& \sum_{c} Z_{c u} \leq 1, \quad \forall u \in \mathcal{U}, \\
& \sum_{u} X_{c u b z}=1, \quad \forall(c, b, z) \in \mathcal{C} \times \mathcal{B} \times \mathcal{Z}, \\
& Y_{u z}=\sum_{c b} X_{c u b z} \leq 1, \quad \forall(u, z) \in \mathcal{U} \times \mathcal{Z}, \\
& X_{c u b z}, Y_{u z}, Z_{c u} \in\{0,1\},
\end{aligned}
$$

where the optimization is over the binary variables $X_{c u b z}$, $Y_{u z}$, and $Z_{c u}$ and the notation $\delta($.$) refers to the discrete Dirac$ function which is equal to 1 if its argument is equal to 0 and 0 otherwise. Both the equality constraint ( $2 b)$ and the inequality constraint (2c) are due to system constraint $\mathrm{C}$. The equality constraints (2d) and (2e) correspond to the system constraints $\mathrm{C} 2$ and $\mathrm{C} 3$, respectively.

Using a generic solver for 0-1 mixed integer programs may require a search over the entire feasible space of solutions, i.e., all possible assignments of users to clouds and PZs of the network BSs. The complexity of such method is prohibitive for any reasonably sized system. The next section, instead, presents a more efficient method to solve the problem by constructing the conflict graph in which each vertex represents an association between clouds, users, BSs, and PZs. The paper reformulates the 0-1 mixed integer programming problem (2) as a maximum-weight independent set problem in the conflict graph, which global optimum can be reached using efficient techniques, e.g., [29], [30].

\section{Multi-Cloud Coordinated Scheduling}

This section presents the optimal solution to the optimization problem (2) by introducing the conflict graph and reformulating the problem as a maximum-weight independent set problem. The corresponding solution is naturally centralized, and the computation must be carried at the central cloud connecting all the clouds $c \in \mathcal{C}$.

\section{A. Conflict Graph Construction}

Define $\mathcal{A}=\mathcal{C} \times \mathcal{U} \times \mathcal{B} \times \mathcal{Z}$ as the set of all associations between clouds, users, BSs, and PZs, i.e., each element $a \in \mathcal{A}$ represents the association of one user to a cloud and a PZ in one of the connected BSs frame. For each association $a=(c, u, b, z) \in \mathcal{A}$, let $\pi(a)$ be the benefit of such association defined as $\pi(a)=\pi_{c u b z}$. Let $\varphi_{c}$ be the cloud association function that maps each element from the set $\mathcal{A}$ to the corresponding cloud in the set $\mathcal{C}$. In other words, for $a=(c, u, b, z) \in \mathcal{A}, \varphi_{c}(a)=c$. Likewise, let $\varphi_{u}, \varphi_{b}$, and $\varphi_{z}$ be the association functions mapping each element $a=(c, u, b, z) \in \mathcal{A}$ to the set of users $\mathcal{U}$ (i.e., $\varphi_{u}(a)=u$ ), to the set of BSs $\mathcal{B}$ (i.e., $\varphi_{b}(a)=b$ ), and to the set of PZs (i.e., $\left.\varphi_{z}(a)=z\right)$, respectively.

The conflict graph $\mathcal{G}(\mathcal{V}, \mathcal{E})$ is an undirected graph in which each vertex represents an association of cloud, user, BS and PZ. Each edge between vertices represents a conflict between the two corresponding associations. Therefore, the conflict graph can be constructed by generating a vertex $v \in \mathcal{V}$ for each association $a \in \mathcal{A}$. Vertices $v$ and $v^{\prime}$ are conflicting vertices, and thus connected by an edge in $\mathcal{E}$ if one of the following connectivity conditions (CC) is true:

- CC1: $\delta\left(\varphi_{u}(v)-\varphi_{u}\left(v^{\prime}\right)\right)\left(1-\delta\left(\varphi_{c}(v)-\varphi_{c}\left(v^{\prime}\right)\right)\right)=1$.

- CC2: $\left(\varphi_{c}(v), \varphi_{b}(v), \varphi_{z}(v)\right)=\left(\varphi_{c}\left(v^{\prime}\right), \varphi_{b}\left(v^{\prime}\right), \varphi_{z}\left(v^{\prime}\right)\right)$.

- CC3: $\delta\left(\varphi_{u}(v)-\varphi_{u}\left(v^{\prime}\right)\right) \delta\left(\varphi_{z}(v)-\varphi_{z}\left(v^{\prime}\right)\right)=1$.

An independent set in the graph $\mathcal{G}(\mathcal{V}, \mathcal{E})$ is a set of vertices such as no edge exists between any pair of vertices in the set. In other words, an independent set is a set of vertices such that no two vertices are in conflict. The connectivity constraint $\mathrm{CC} 1$ corresponds to a violation of the system constraint $\mathrm{C} 1$ as it describes that two vertices are conflicting if the same user is scheduled to different clouds. The connectivity constraint $\mathrm{CC} 2$ partially illustrates the system constraint $\mathrm{C} 2$, as it implies that each PZ should be associated with at most one user (not exactly one user as stated in the original system constraint). With the additional constraint (see Theorem 1 below) about the size of the independent set, $\mathrm{CC} 2$ becomes equivalent to $\mathrm{C} 2$. Finally, the edge creation condition CC3 correctly translates a violation of the system constraint $\mathrm{C} 3$.

Figure 3 illustrates an example of the conflict graph in a multi-cloud system composed of $C=2$ clouds, $B=2 \mathrm{BSs}$ per cloud, $Z=2$ PZs per BS and $U=4$ users. Vertices, in this example, are labelled $c u b z$, where $c, u, b$ and $z$ represent 


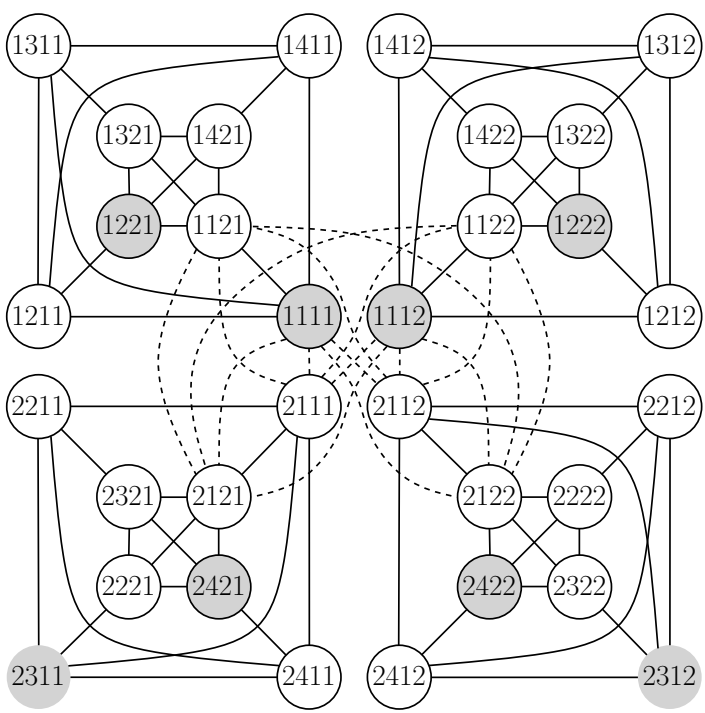

Fig. 3. Example of the conflict graph for a network composed of 2 clouds, 2 BSs per cloud, 2 PZs per BS and a total of 4 users. Intra-cloud connection are plotted in solid lines. Inter-cloud connections are illustrated only for user 1 in dashed lines.

the indices of clouds, users, BSs, and PZs, respectively. In this example, $Z_{\text {tot }}=C B Z=8$. As shown in Figure 3, each independent set of size $Z_{\text {tot }}$ can be written in the following form:

1) $\{1 a 11,1 a 12,1 b 21,1 b 22,2 c 11,2 c 12,2 d 21,2 d 22\}$

2) $\{1 a 11,1 a 12,1 b 21,1 b 22,2 c 11,2 d 12,2 d 21,2 c 22\}$

3) $\{1 a 11,1 b 12,1 b 21,1 a 22,2 c 11,2 c 12,2 d 21,2 d 22\}$

4) $\{1 a 11,1 b 12,1 b 21,1 a 22,2 c 11,2 d 12,2 d 21,2 c 22\}$,

where $a, b, c$, and $d \in\{1,2,3,4\}$ with $a \neq b \neq c \neq d$. For example, replacing $(a, b, c, d)$ in $\{1 a 11,1 a 12,1 b 21,1 b 22$, $2 c 11,2 c 12,2 d 21,2 d 22\}$ by $(1,2,3,4)$ gives the independent set shown in gray in Figure 3, which is a set of non-connected vertices of size $Z_{\text {tot }}=8$. The $4 !=24$ distinct permutations of $(a, b, c, d)$ eventually result in $4 ! \times 4=96$ independent sets of size $Z_{\text {tot }}$ in total.

\section{B. Scheduling Solution}

Consider the conflict graph $\mathcal{G}(\mathcal{V}, \mathcal{E})$ constructed above and let $\mathcal{I}$ be the set of all independent set of vertices of size $Z_{\text {tot }}=$ $C B Z$. The following theorem characterises the solution of the optimization problem (2).

Theorem 1. The global optimal solution to the scheduling problem in multi-cloud network (2) is the maximum-weight independent set among the independent sets of size $Z_{\text {tot }}$ in the conflict graph, where the weight of each vertex $v \in \mathcal{V}$ is given by:

$$
w(v)=\pi(v) .
$$

In other words, the optimal solution of the scheduling problem (2) can be expressed as:

$$
I^{*}=\arg \max _{I \in \mathcal{I}} \sum_{v \in I} w(v) .
$$

Proof: A sketch of the proof goes as follows. The optimization problem (2) is first reformulated as a search over the set of feasible schedules. Further, a one to one mapping

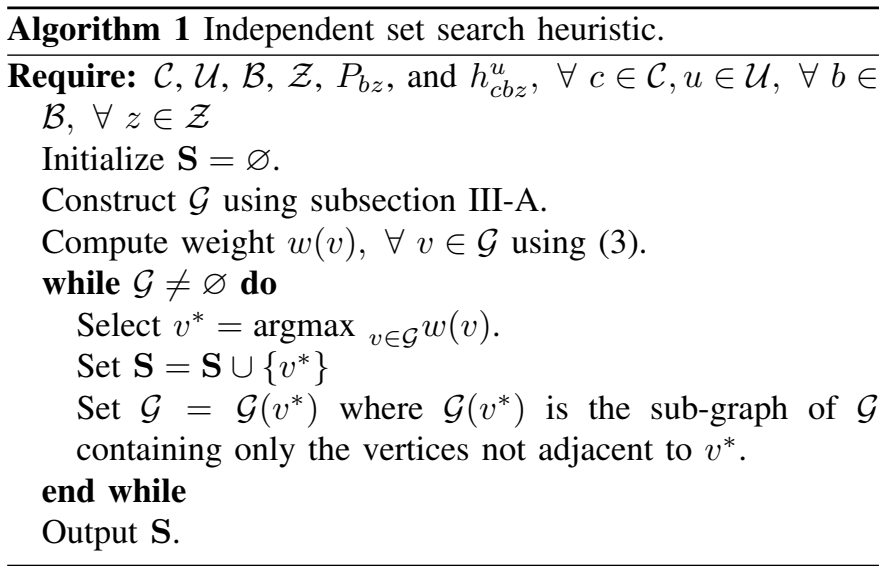

between the possible schedules and the set of independent sets of size $Z_{t o t}$ in the conflict graph is established. Finally, showing that the weight of each independent set is the objective function of (2) indicates that the optimal solution is the maximum-weight independent set, which concludes the proof. A complete proof can be found in Appendix A.

\section{Complexity Analysis and Heuristic Algorithm}

In graph theory context, an independent set is a set in which each two vertices are not adjacent. The maximumweight independent set problem is the problem of finding, in a weighted graph, the independent set(s) with the maximum weight where the weight of the set is defined as the sum of the individual weights of vertices belonging to the set. Maximumweight independent set problems are well-known NP-hard problems. However, they can be solved efficiently, e.g., [29], [30]. Therefore, the complexity of the proposed solution can be written as $\mathbb{C}_{\mathrm{cen}}^{\mathrm{opt}}=\alpha^{C B Z U}$, where $1<\alpha \leq 2$ is a constant that depends on the applied algorithm, e.g., $\alpha=1.21$ for [30]. Moreover, several approximate [31] and polynomial time [32] methods produce satisfactory results, in general. This subsection presents a heuristic, yet simple, algorithm which discovers a maximal ${ }^{1}$ weight independent set.

To solve the maximum-weight independent set problem in linear time with the size of the graph, a simple procedure is to sequentially select nodes with largest weights. First, construct the graph $\mathcal{G}$. The idea here is to sequentially update the independent set $\mathbf{S}$ by adding the vertex with the highest weight at each step. Then, the graph is updated by removing all vertices adjacent to the selected vertex, so as to guarantee that the connectivity constraints $\mathrm{CC} 1, \mathrm{CC} 2$, and $\mathrm{C} 3$ are satisfied. The process is repeated until the graph becomes empty. The steps of the heuristic are summarized in Algorithm 1.

\section{Distributed CoORdinated Scheduling}

The previous section assumes the presence of a central cloud that is responsible for computing the scheduling policy in a centralized fashion, which may not always be feasible from a computational complexity perspective. This section instead

\footnotetext{
${ }^{1} \mathrm{~A}$ maximal independent set is a set that is no longer independent if any node is added to it. The maximum independent set is the maximum of all such maximal sets.
} 


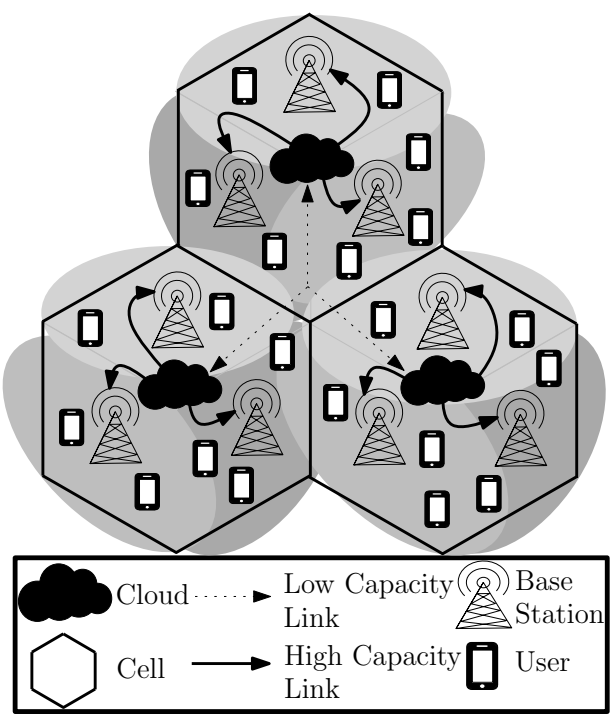

Fig. 4. Cloud enabled network composed 3 cells, each containing 3 base stations and 7 users.

considers the multi-CRAN in which clouds are interconnected through low capacity links, as shown in Figure 4. In contrast to the centralized system in Figure 1, the joint scheduling is now performed under the constraint that each cloud $c \in \mathcal{C}$ has partial access to the network parameters. In particular, cloud $c \in \mathcal{C}$ has knowledge of the its channel gains only, i.e., $h_{c b z}^{u}, \forall(u, b, z) \in \mathcal{U} \times \mathcal{B} \times \mathcal{Z}$. The distributed joint scheduling problem becomes the one of scheduling users to clouds and PZs in the connected BSs, by only allowing a reasonable amount of information exchange among the clouds.

Remark 1. Note that exchanging all the network parameters, i.e., $h_{c b z}^{u}, \forall(c, u, b, z) \in \mathcal{C} \times \mathcal{U} \times \mathcal{B} \times \mathcal{Z}$, constructing the conflict graph at each cloud and solving the maximumweight independent set may solve the optimization problem (2). However, such solution not only requires a considerable amount of backhaul communication (exchange of $C U B Z$ complex variable), but also a waste of computation resources as the $C$ clouds solve the same problem.

The first part of this section provides the optimal distributed coordinated scheduling. In other words, this part characterizes the solution of the optimization problem (2) by solving the maximum-weight independent set in the conflict graph in a distributed fashion. The second part of this section provides a heuristic, low complexity, distributed solution for problem (2).

\section{A. Optimal Distributed Coordinated Scheduling}

To solve the scheduling problem (2) in a distributed fashion, the paper proposes a distributed method to resolve the maximum-weight independent set using the particular structure of the conflict graph. Before describing the steps of the algorithm, the section first introduces the local scheduling graph $\mathcal{G}_{c}\left(\mathcal{U}_{c}\right)$ for an arbitrary cloud $c \in \mathcal{C}$ and its set of scheduled users $c$, called $\mathcal{U}_{c} \subseteq \mathcal{U}$.

Let the reduced set of association of cloud $c$ be defined as $\hat{\mathcal{A}}=c \times \mathcal{U}_{c} \times \mathcal{B} \times \mathcal{Z}$. This set represents all associations cloud

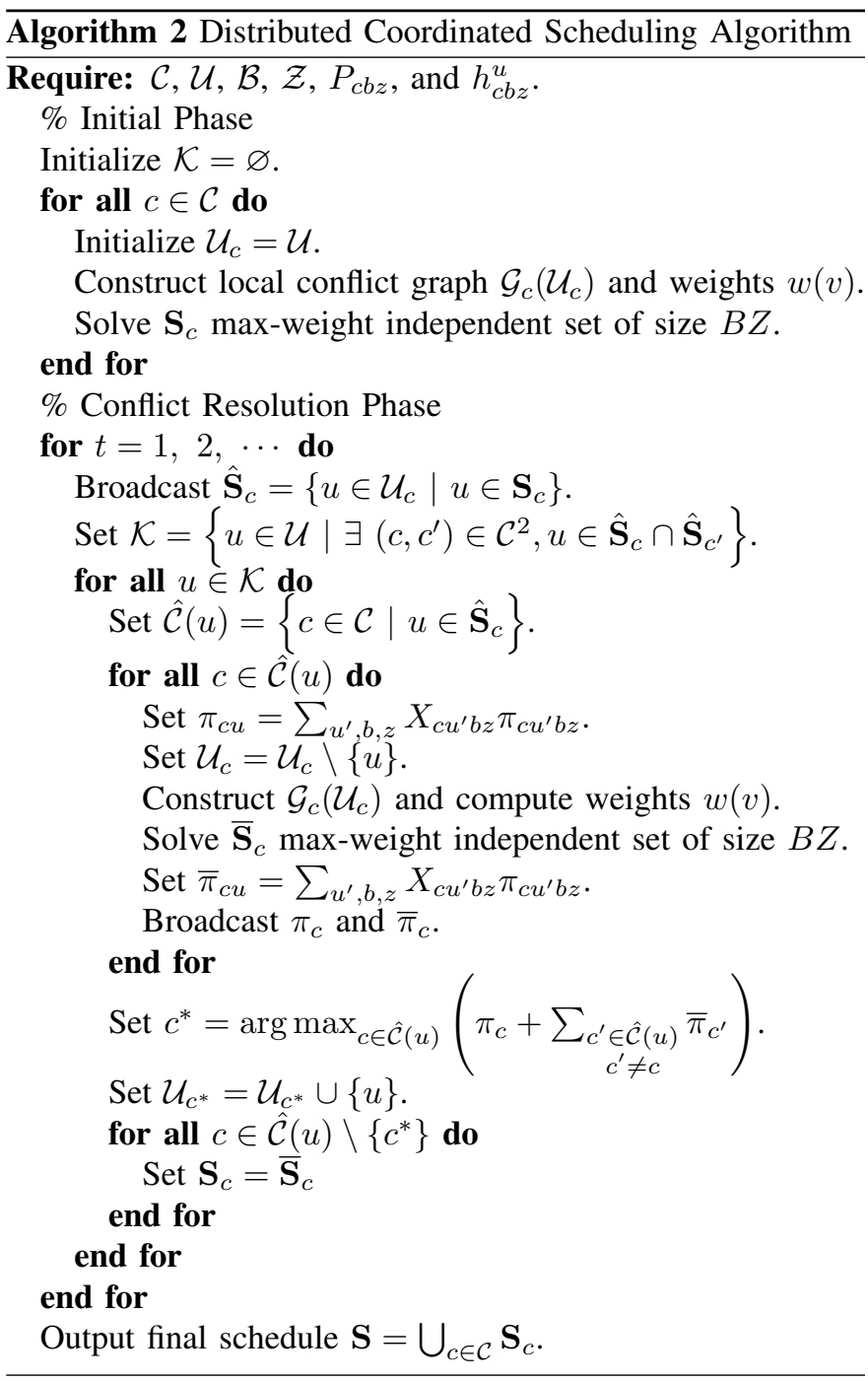

$c$ can perform when it is allowed to schedule users in the set $\mathcal{U}_{c}$. Note that all the benefits of the associations $\hat{a} \in \hat{\mathcal{A}}$ can be computed locally at cloud $c$ since all the needed complex channel gains $h_{c b z}^{u}, \forall(u, b, z) \in \mathcal{U} \times \mathcal{B} \times \mathcal{Z}$ and power levels $P_{c b z}, \forall(b, z) \in \mathcal{B} \times \mathcal{Z}$ are locally available. The local conflict graph $\mathcal{G}_{c}\left(\mathcal{U}_{c}\right)$ is constructed in a similar manner as the conflict graph $\mathcal{G}$ except that it only considers associations $\hat{a} \in \hat{\mathcal{A}}$ in the vertex generation step. Hence, instead of containing $C U B Z$ vertex, the local conflict graph contains $U_{c} B Z$ where $U_{c}=$ $\left|\mathcal{U}_{c}\right|$. The vertex connectivity conditions are the same as for the conflict graph.

The algorithm is composed of two phases, namely, the initialization and conflict resolution phases. In an initial phase, each cloud generates its local conflict graph and solves the maximum-weight independent set of size $B Z$. Each cloud communicates its scheduled users with the remaining clouds. It is worth mentioning that only the scheduled users are shared and not the complete information about the schedule (i.e., the PZs and the BSs in which they are scheduled).

After the initial phase, a conflict resolving step takes place. In this stage, users that are scheduled to multiple clouds are assigned to the cloud that generates the highest sumbenefit of scheduling that user across its multiple BSs and 
PZs. Clouds $c^{\prime}$ that fail to have the maximum benefit are not allowed to schedule that user in the subsequent phases of the algorithm. Clouds $c^{\prime}$, therefore, remove that user from their set of authorized users. The new graph is then constructed, and the maximum weight clique is subsequently solved. This process is repeated until all users are assigned to at most one single cloud. The steps of the algorithm are summarized in Algorithm 2.

The following theorem characterizes the distributed solution reached by Algorithm 2:

Theorem 2. Algorithm 2 converges to the optimal solution of the centralized coordinated scheduling optimization problem (2) in at most $C(U-B)$ iterations.

Proof: To show that the distributed solution reached by Algorithm 2 is the optimal solution to the scheduling problem (2), we first show that solving the maximum-weight independent set locally yields the optimal solution to (2), whenever the local and the global approaches start with users assigned to the same cloud. Afterward, we show that Algorithm 2 assigns users to clouds that coincide with the cloud assignment found through the optimal solution. To finish the proof, we show that the running time of the algorithm is bounded. A complete proof of the theorem can be found in Appendix B.

\section{B. Heuristic Distributed Coordinated Scheduling}

In this section, a heuristic, low-complexity, distributed solution is presented. The algorithm follows the same steps as Algorithm 2, except in the way of updating the local maximum-weight independent set at each cloud. While Algorithm 2 recomputes the new graph and the maximum-weight independent set for each user in conflict, the proposed lowcomplexity distributed solution updates the solution obtained in the previous round. In other words, instead of generating the new graph and recomputing the maximum-weight independent set at each step, the heuristic algorithm updates the maximumweight clique obtained previously by removing vertices in conflict and adding new vertices, which simplifies the computational complexity.

To explicitly define the maximum-weight independent set update strategy, first, define $\mathbf{S}_{c}$ as the schedule obtained by solving the maximum-weight independent set, and $\mathbf{S}_{c}^{u} \subset \mathbf{S}_{c}$ as the set of vertices of user $u$ scheduled in cloud $c$. Further, let $\mathcal{V}_{c}\left(\mathbf{S}_{c}\right)$ be the set of vertices in the local conflict graph $\mathcal{G}_{c}\left(\mathcal{U}_{c}\right)$ that are not connected to any vertex in $\mathbf{S}_{c}$. Note that the vertices in $\mathcal{V}_{c}\left(\mathbf{S}_{c}\right)$ are combinable with the previous schedule $\mathbf{S}_{c}$ since they are not connected to any vertex in the schedule.

The low-complexity distributed algorithm follows the same steps in the initial phase as Algorithm 2. In the conflict resolution phase, users that are scheduled to multiple clouds are assigned to the one with the highest sum-benefit. The remaining clouds remove the associations containing the user, i.e., vertices in $\mathbf{S}_{c}^{u}$, from their schedule. Afterward, they update their local conflict graph to only keep the vertices $\mathcal{V}_{c}\left(\mathbf{S}_{c}\right)$ that are not connected to all vertices previously selected in the schedule $\mathbf{S}_{c} \backslash \mathbf{S}_{c}^{u}$. The maximum-weight independent set of size $\left|\mathbf{S}_{c}^{u}\right|$ is then computed and appended to $\mathbf{S}_{c}$ to produce the

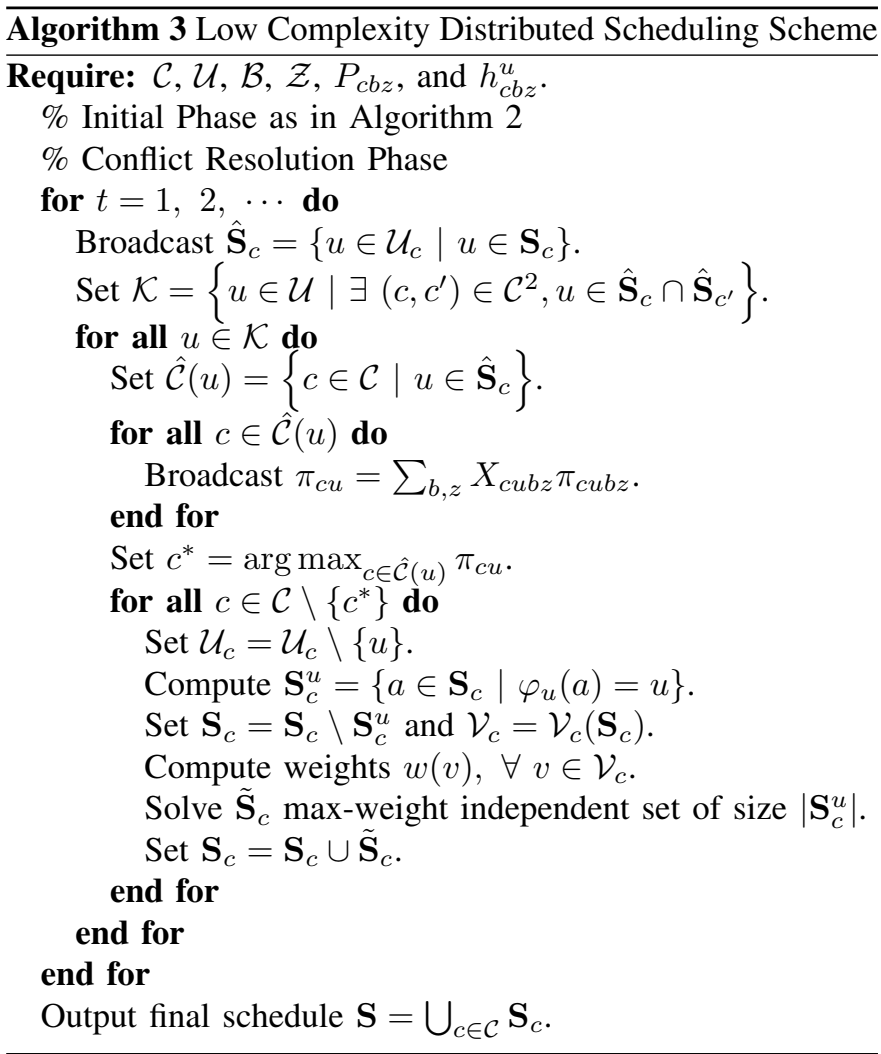

schedule. The process is repeated until all users are assigned to at most one single cloud. The steps of the algorithm are summarized in Algorithm 3.

Corollary 1. Algorithm 3 converges to a feasible solution of the centralized coordinated scheduling optimization problem (2) in at most $U$ iterations.

Proof: To prove this corollary, it is sufficient to show that Algorithm 3 converges. Showing that the outputted schedule is a feasible one concludes the proof. A complete proof can be found in Appendix C.

\section{Complexity Analysis}

This subsection computes the worst case computational complexity of the optimal and heuristic distributed algorithms. It further compares such complexities to the complexity of the centralized solution proposed in Section III. Moreover, the subsection shows that the complexity of the heuristic distributed algorithm is negligible as compared to the optimal distributed algorithm, as the number of clouds increases. Section VI further compares the performance of both algorithms as the number of clouds increases, so as to show that the degradation in performance is limited especially for the high complexity gain.

As shown in Algorithm 2, each cloud solves a maximum weight independent set at each step of the algorithm in which it has conflicts with other clouds. The size of the scheduling graph of the $c$-th cloud is $C B Z\left|\mathcal{U}_{c}\right|$. Given the result in Theorem 2, each cloud experiences $U-B$ conflict in the worst case. Therefore, the total complexity $\mathbb{C}_{\text {dis }}^{\text {opt }}$ per cloud can 
be written as:

$$
\begin{aligned}
\mathbb{C}_{\mathrm{dis}}^{\mathrm{opt}} & =\sum_{\left|\mathcal{U}_{c}\right|=U-B}^{U} \alpha^{C B Z\left|\mathcal{U}_{c}\right|}=\alpha^{C B Z U} \frac{\alpha^{-C B^{2} Z}-\alpha^{C B Z}}{1-\alpha^{C B Z}} \\
& =\mathbb{C}_{\mathrm{cen}}^{\mathrm{opt}} \frac{\alpha^{-C B^{2} Z}-\alpha^{C B Z}}{1-\alpha^{C B Z}}
\end{aligned}
$$

where $1<\alpha \leq 2$ is a constant that depends on the algorithm utilized in solving the maximum weight independent set problem. The important conclusion from (5) is that the complexity of the distributed solution approaches the complexity of the centralized one as the number of base-stations and/or PZs increases. This can be explained by the fact that for more and more available resources, there are more scheduling opportunities, and hence less conflicts.

The analysis of the low complexity distributed algorithm follows the same lines as the optimal one, except that the total number of conflicts experienced by all clouds is bounded by $U$ according to Corollary 1 . Therefore, assuming each cloud experience $[U / C\rfloor$ conflicts, the complexity per cloud can be experienced as follows:

$$
\begin{aligned}
\mathbb{C}_{\mathrm{dis}}^{\text {heu }} & =\sum_{\left|\mathcal{U}_{c}\right|=\lfloor U / C\rfloor}^{U} \alpha^{C B Z\left|\mathcal{U}_{c}\right|}=\alpha^{C B Z U} \frac{\alpha^{B Z U(1-C)}-\alpha^{C B Z}}{1-\alpha^{C B Z}} \\
& =\mathbb{C}_{\mathrm{cen}}^{\text {opt }} \frac{\alpha^{B Z U(1-C)}-\alpha^{C B Z}}{1-\alpha^{C B Z}}
\end{aligned}
$$

where $1<\alpha \leq 2$ is a constant that depends on the utilized algorithm. It is worth mentioning that unlike the optimal distributed algorithm, the number of users in the network plays a role on the complexity of the heuristic. In fact, the heuristic algorithm considers conflicts of users instead of the conflicts of clouds considered in the optimal solution. To further show how the heuristic presents a serious complexity gain over the optimal solution, the section proposes comparing relative complexity between the optimal and heuristic solutions. The relative complexity is given by the following expression:

$$
\frac{\mathbb{C}_{\mathrm{dis}}^{\text {heu }}}{\mathbb{C}_{\mathrm{dis}}^{\text {opt }}}=\frac{\alpha^{B Z U(1-C)}-\alpha^{C B Z}}{\alpha^{-C B^{2} Z}-\alpha^{C B Z}}
$$

The above expression reveals that the heuristic solution provides a complexity gain under the assumption that $C(U-B) \geq$ $U$ which holds with high probability for any reasonably sized network. Such complexity simplification comes, however, at the expense of a degradation in the performance as the number of clouds increases, as the simulations section suggests later.

\section{EXTREMES IN COORDINATION SCHEMES}

The two extremes in coordination schemes are presented in this section. The fully coordinated system, also known as the signal-level coordinated system, requires a substantial amount of backhaul communication to share all the data streams between the BSs. On the other hand, scheduling-level coordination requires low capacity links to connect all BSs to clouds, as clouds become responsible for determining the scheduling policy of the network only. Although more practical to implement from backhaul requirements perspective, scheduling-level coordination comes at the expense of performance degradation. This section considers the two scheduling policy extremes, i.e., either scheduling-level or signal-level coordination problems. These two allocation problems are separately considered in the literature, e.g., [7]-[12] for the single cloud setting. This part next shows that the proposed graph theoretical framework developed earlier in this paper can be alternatively used to globally solve the generalization of the problems to the multi-cloud setting of this paper. In other words, the scheduling problem in each case can be solved using similar techniques to the one used in solving the original hybrid scheduling problem.

\section{A. Signal-Level Coordination}

For signal-level coordinated systems, all the data streams of users are shared among the BSs across the network. Hence, a user can be scheduled to many BSs in different clouds. The scheduling problem becomes the one of assigning users to clouds and scheduling them to PZs in each BS frame under the following practical constraints.

- Each PZ should be allocated to exactly one user.

- Each user cannot be served by the same PZ across different BSs.

Following an analysis similar to the one in Section III, the scheduling problem can be formulated as a 0-1 mixed integer programming as follows:

$$
\begin{aligned}
\max & \sum_{c, u, b, z} \pi_{c u b z} X_{c u b z} \\
\text { s.t. } & \sum_{u} X_{c u b z}=1, \quad \forall(c, b, z) \in \mathcal{C} \times \mathcal{B} \times \mathcal{Z}, \\
& \sum_{c b} X_{c u b z} \leq 1, \quad \forall(u, z) \in \mathcal{U} \times \mathcal{Z}, \\
& X_{c u b z} \in\{0,1\}, \forall(c, u, b, z) \in \mathcal{C} \times \mathcal{U} \times \mathcal{B} \times \mathcal{Z},
\end{aligned}
$$

where the optimization is over the binary variable $X_{c u b z}$, and where equations (8b) and (8c) correspond to the first and second system constraints, respectively.

Construct a graph similar to the one constructed in Section III, except using the connectivity constraints $\mathrm{CC} 2$ and CC3 only. Such graph, denoted by $\mathcal{G}^{\prime}\left(\mathcal{V}^{\prime}, \mathcal{E}^{\prime}\right)$, is called here the reduced conflict graph. The following lemma provides the optimal solution to the optimization problem (8).

Lemma 1. The optimal solution to the scheduling problem in signal-level coordinated cloud-enabled network (8) is the maximum-weight independent set of size $C B Z$ in the reduced conflict graph which is constructed in a similar manner as the conflict graph but using only connectivity constraint CC2 and CC3.

Proof: A sketch of the proof goes as follows. The constraints (8b), (8c) and (8d) of the optimization problem (8) are similar to constraints (2d), (2e) and (2f), respectively. Therefore, this lemma can be proved using similar steps of Theorem 1, except by considering the reduced conflict graph $\mathcal{G}^{\prime}\left(\mathcal{V}^{\prime}, \mathcal{E}^{\prime}\right)$ only. A complete proof can be found in Appendix D.

\section{B. Scheduling-Level Coordination}

In scheduling-level coordinated CRAN, the cloud is only responsible for scheduling users to BSs and PZs and synchronizing the transmit frames across the various BSs. In such 
TABLE I

SYSTEM MODEL PARAMETERS

\begin{tabular}{|c|c|}
\hline Cellular Layout & Hexagonal \\
\hline Cell-to-Cell Distance & 500 meters \\
\hline Channel Model & SUI-3 Terrain type B \\
\hline Channel Estimation & Perfect \\
\hline High Power & $-42.60 \mathrm{dBm} / \mathrm{Hz}$ \\
\hline Background Noise Power & $-168.60 \mathrm{dBm} / \mathrm{Hz}$ \\
\hline SINR Gap $\Gamma$ & $0 \mathrm{~dB}$ \\
\hline Bandwidth & $10 \mathrm{MHz}$ \\
\hline
\end{tabular}

coordinated systems, the scheduling problem is the one of assigning users to BSs and PZs under the following system constraints:

- Each user can connect at most to one BS but possibly to many PZs in that BS.

- Each PZ should be allocated to exactly one user.

The scheduling problem can, then, be formulated as follows:

$$
\begin{aligned}
\max & \sum_{c, u, b, z} \pi_{c u b z} X_{c u b z} \\
\text { s.t. } & Y_{c u b}=\min \left(\sum_{z} X_{c u b z}, 1\right), \quad \forall(c, u, b), \\
& \sum_{c, b} Y_{c u b} \leq 1, \quad \forall u \in \mathcal{U}, \\
& \sum_{u} X_{c u b z}=1, \quad \forall(c, b, z) \in \mathcal{C} \times \mathcal{B} \times \mathcal{Z}, \\
& X_{c u b z}, Y_{c u b} \in\{0,1\}, \quad \forall(c, u, b, z),
\end{aligned}
$$

where the optimization is over the binary variables $X_{c u b z}$ and $Y_{c u b}$, where the constraints in (9b) and (9c) correspond to first system constraint, and where the equality constraint in (9d) corresponds to the second system constraint.

Construct the scheduling conflict graph $\mathcal{G}^{\prime \prime}\left(\mathcal{V}^{\prime \prime}, \mathcal{E}^{\prime \prime}\right)$ by generating a vertex $v \in \mathcal{V}^{\prime \prime}$ for each association $a \in \mathcal{A}$. Vertices $v$ and $v^{\prime}$ are conflicting vertices, and thus connected by an edge in $\mathcal{E}^{\prime \prime}$ if one of the following connectivity conditions is true:

- $\delta\left(\varphi_{u}(v)-\varphi_{u}\left(v^{\prime}\right)\right)\left(1-\delta\left(\varphi_{c}(v)-\varphi_{c}\left(v^{\prime}\right)\right)\right)=1$.

- $\left(\varphi_{c}(v), \varphi_{b}(v), \varphi_{z}(v)\right)=\left(\varphi_{c}\left(v^{\prime}\right), \varphi_{b}\left(v^{\prime}\right), \varphi_{z}\left(v^{\prime}\right)\right)$.

- $\delta\left(\varphi_{u}(v)-\varphi_{u}\left(v^{\prime}\right)\right)\left(1-\delta\left(\varphi_{b}(v)-\varphi_{b}\left(v^{\prime}\right)\right)\right)=1$.

The following proposition characterizes the solution of the scheduling problem in scheduling-level coordinated CRANs:

Proposition 1. The optimal solution to the optimization problem (9) is the maximum-weight independent set of size CBZ in the scheduling conflict graph.

The proof of this result is omitted as it mirrors the steps used in proving Theorem 1.

\section{Simulation Results}

The performance of the proposed scheduling schemes is shown in this section in the downlink of a cloud-radio access network, similar to Figure 4. For illustration purposes, the simulations focus on the sum-rate maximization problem, i.e., $\pi_{c u b z}=\log _{2}\left(1+\operatorname{SINR}_{c b z}^{u}\right)$. In these simulations, the cell size is set to 500 meters and users are uniformly placed within each

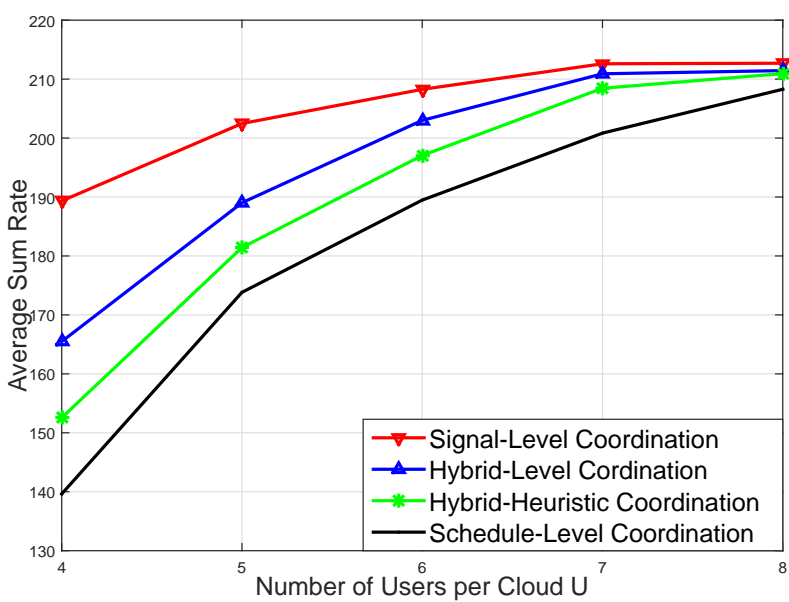

Fig. 5. Sum-rate in bps/Hz versus number of users $U$. Number of clouds is $C=3$ with $B=3$ base-stations per cloud, and $Z=5$ power-zones per BS's transmit frame.

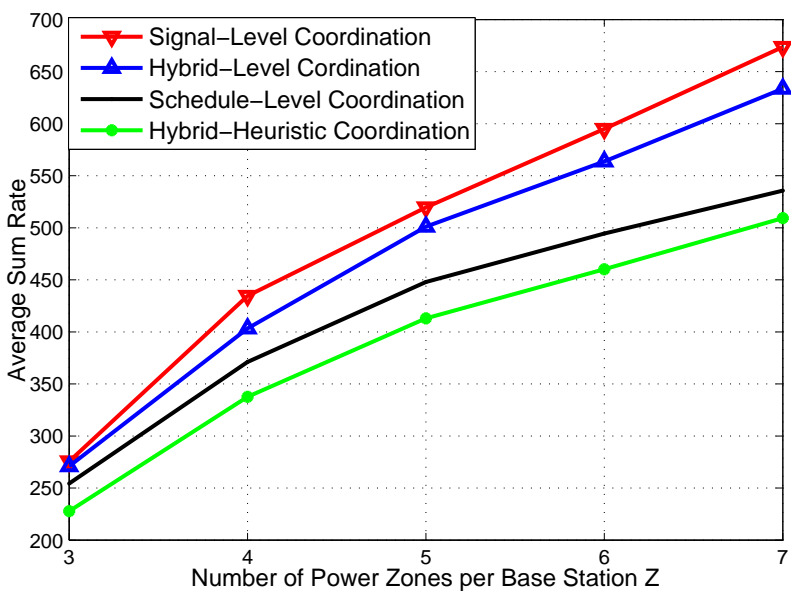

Fig. 6. Sum-rate in bps/Hz versus number of power-zones $Z$ per BS for a large system. The network is composed of 7 clouds, each containing $B=3$ base-stations. The network serves a total of 56 users.

cell. The number of clouds, users, base-stations per cloud and power-zone per base-station frame change in each figure in order to quantify the gain in various scenarios. Simulations parameters are displayed in Table I. It is crucial to highlight that both the centralized and the distributed optimal algorithms described in Section III and Section IV.A provide the exact same solution denoted by "Hybrid-level coordination". All simulation are carried out on a Windows 10 laptop with 2.4 $\mathrm{GHz}$ Intel Core i7 processor and $8 \mathrm{~GB} 1600 \mathrm{MHz}$ DDR3 RAM.

Figure 5 plots the sum-rate in $\mathrm{bps} / \mathrm{Hz}$ versus the number of users $U$ for a CRAN composed of $C=3$ clouds, $B=3$ base-stations per cloud, and $Z=5$ power-zones per BS's transmit frame. The proposed hybrid coordination policy provides a significant gain against the schedulinglevel coordinated system for a small number of users. As the number of users increases in the system, the different strategies performs the same. This can be explained by the fact that as the number of users in the network increases, the probability that different users have the maximum payoff in various PZs across the network increases, which results in scheduling different users in different PZs and thus the 


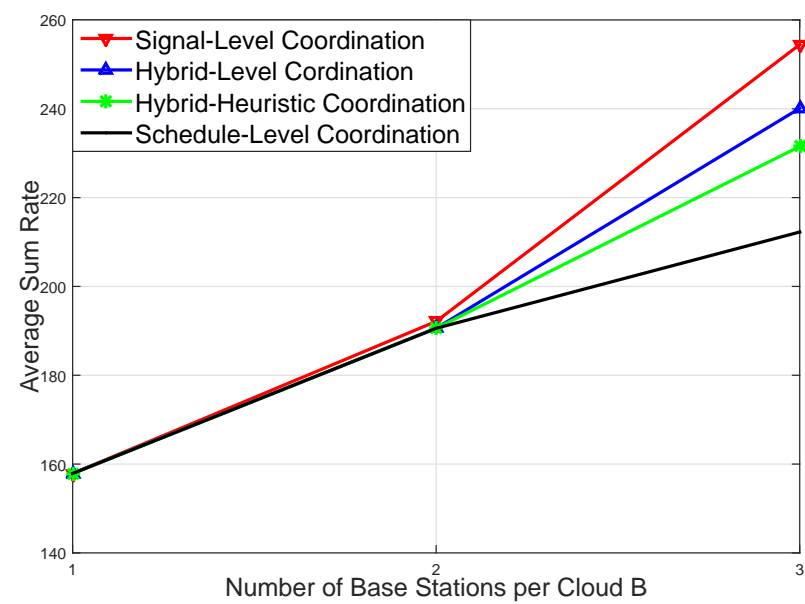

Fig. 7. Sum-rate in bps/Hz versus number of base-stations $B$ per cloud. Number of clouds is $C=3$ with $Z=5$ power-zones per BS's transmit frame, and $U=24$ users.

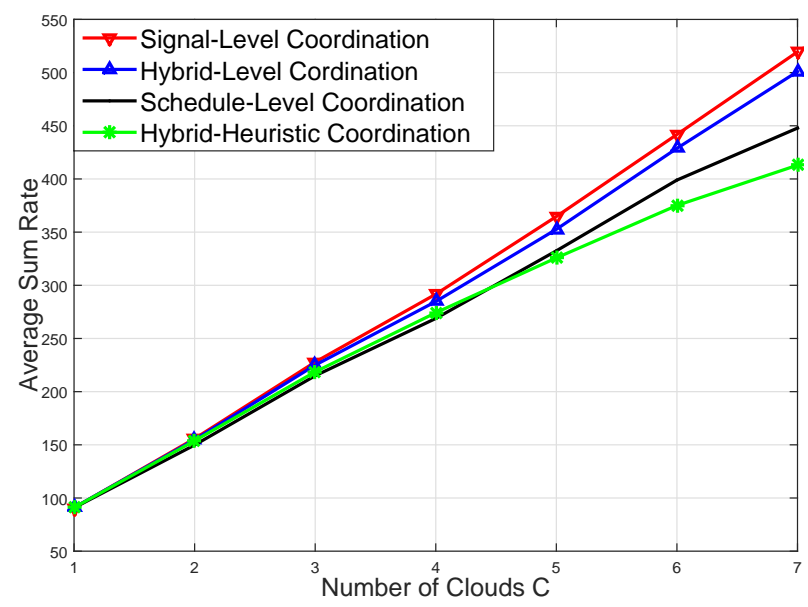

Fig. 8. Sum-rate in bps/Hz versus number of clouds $C$. Number of basestations is $B=3$ per cloud, with 5 power-zones per BS's transmit frame, and $U=8$ users per cloud.

different scheduling policies provide similar performance. The performance of the distributed heuristic approaches the one of the optimal scheduling as the number of users increases. This can be explained by the fact that for a large number of users, the probability that a user is scheduled to more than one cloud decreases, which decreases the conflict among clouds and the likelihood of scheduling user to the wrong cloud.

Figure 6 plots the sum-rate in $\mathrm{bps} / \mathrm{Hz}$ versus the number of power-zones $Z$ per BS for a large network comprising $C=7$ clouds, $B=3$ base-stations, and $U=56$ users. The figure reveals that for a small number of PZs per BS, all scheduling policies have equivalent performance. As the number of PZs per BS increases, the gap between the different coordinated systems increases. In fact, as the number of PZs increases, the ratio of users per PZ decreases and thus the role of the cloud as a scheduling entity becomes more pronounced. The figure further shows that the proposed heuristic degrades in performance for a large number of clouds due to the suboptimal allocation of users to clouds. The effect is further illustrated in Figure 8.

Figure 7 plots sum-rate in bps/Hz versus the number of base-stations $B$ per cloud for a network comprising $C=3$

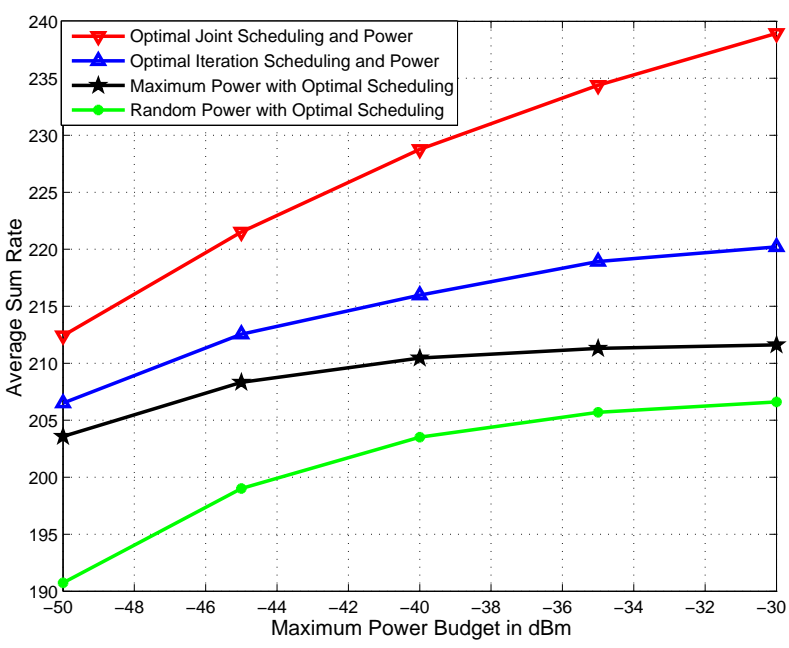

Fig. 9. Sum-rate in $\mathrm{bps} / \mathrm{Hz}$ versus the maximum power per $\mathrm{PZ}$. The networks in composed of $C=3$ clouds, each containing $B=3$ BSs with 5 PZs. The network serves a total number of $U=24$ users.

clouds, $Z=5$ power-zones per BS's transmit frame, and $U=24$ users. For a small number of BSs, all the policies are equivalent and provide the same gain. However, as this number increases, the higher the level of coordination is, the more scheduling opportunities it offers. This explains the difference in performance as $B$ increases. We can see that our hybrid coordination provides a gain up to $13 \%$ as compared to the scheduling-level coordinated network, for a degradation up to $6 \%$ as compared to the signal-level coordination.

Figure 8 plots the sum-rate in $\mathrm{bps} / \mathrm{Hz}$ versus the number of clouds $C$ for a network comprising $B=3$ base-stations per cloud, $Z=5$ power-zones per BS's transmit frame, and $U=8$ users per cloud. Again, our hybrid coordination provides a gain up to $12 \%$ as compared with the scheduling-level coordination, for a negligible degradation up to $4 \%$ against the signal-level coordinated system. For a large number of clouds, the performance of the distributed heuristic degrades. This can be explained by the fact that for a large number of clouds, the probability that multiple clouds are in conflict for the same user increases, which increases the probability of scheduling users to the wrong cloud; thereby resulting in a performance degradation.

Finally, Figure 9 illustrates the performance in sum-rate in $\mathrm{bps} / \mathrm{Hz}$ of the proposed hybrid scheduling policy with different power adaptation techniques against the maximum power per PZ, for a CRAN composed of $C=3$ clouds, each containing $B=3 \mathrm{BSs}$ with $5 \mathrm{PZs}$. The figure compares the achieved throughput by the joint and modular power adaptation approaches and the one obtained by the random and maximum power assignment with optimal scheduling. The random power allocation scheme associates a random power fraction between 0.85 and 1 of the total energy budget. The figure shows that the joint scheduling technique offers an appreciable gain for a high allowable power which emphasizes the interference mitigation role of the cloud. However, for a small maximum allowable power, the modular and maximum power policies provide similar performance for a massive reduction in computation complexity. 


\section{CONCLUSIONS}

This paper considers the hybrid scheduling problem in the downlink of a multi-cloud radio-access network. The paper maximizes a network-wide utility under the practical constraint that each user is scheduled, at most, to a single cloud, but possibly to many BSs within the cloud and can be served by one or more distinct PZs within the BSs frame. The paper proposes a graph theoretical approach to solving the problem by introducing the conflict graph in which each vertex represents an association of cloud, user, BS and PZ. The problem is then reformulated as a maximum-weight independent set problem that can be efficiently solved. The paper further proposes distributed optimal and heuristic solutions to the coordinated scheduling problem. Finally, the paper shows that the optimal solution to the scheduling problem in different levels of system coordination can be obtained as a special case of the more general proposed system. Simulation results suggest that the proposed system architecture provides appreciable gain as compared to the scheduling-level coordinated networks, for a negligible degradation against the signal-level coordination.

\section{APPENDIX A}

\section{ProOF OF THEOREM 1}

To prove the result, the optimization problem (2) is first reformulated as a search over the set of feasible schedules. Further, a one to one mapping between the possible schedules and the set of independent sets of size $Z_{t o t}$ in the conflict graph is highlighted. Showing that the weight of each independent set is the objective function of (2) indicates that the optimal solution is the maximum-weight independent set which concludes the proof.

All possible schedules representing the assignments between clouds, users, BSs and PZs, regardless of the feasibility, can be conveniently represented by the set of all subsets of $\mathcal{A}$, i.e., the power set $\mathcal{P}(\mathcal{A})$ of the set of associations $\mathcal{A}$. Recall that for an association $a=(c, u, b, z)$ in a schedule $\mathcal{S} \subseteq \mathcal{A}$ (i.e., $\mathcal{S} \in \mathcal{P}(\mathcal{A})$ ), the benefit of the association is given by $\pi(a)=\pi_{c u b z}$. The following lemma reformulates the multicloud joint scheduling problem.

Lemma 2. The discrete optimization problem (2) can be written as follows:

$$
\begin{aligned}
& \max _{\mathcal{S} \in \mathcal{P}(\mathcal{A})} \sum_{a \in \mathcal{S}} \pi(a) \\
& \text { s.t. } \mathcal{S} \in \mathcal{F},
\end{aligned}
$$

where $\mathcal{F}$ is the set of feasible schedules defined as follows:

$$
\begin{aligned}
& \mathcal{F}=\left\{\mathcal{S} \in \mathcal{P}(\mathcal{A}) \text { such that } \forall a \neq a^{\prime} \in \mathcal{S}\right. \\
& \delta\left(\varphi_{u}(a)-\varphi_{u}\left(a^{\prime}\right)\right)\left(1-\left(\delta\left(\varphi_{c}(a)-\varphi_{c}\left(a^{\prime}\right)\right)\right)=0,\right. \\
& \left(\varphi_{c}(a), \varphi_{b}(a), \varphi_{z}(a)\right) \neq\left(\varphi_{c}\left(a^{\prime}\right), \varphi_{b}\left(a^{\prime}\right), \varphi_{z}\left(a^{\prime}\right)\right), \\
& \delta\left(\varphi_{u}(a)-\varphi_{u}\left(a^{\prime}\right)\right) \delta\left(\varphi_{z}(a)-\varphi_{z}\left(a^{\prime}\right)\right)=0 \\
& \left.|\mathcal{S}|=Z_{\text {tot }}\right\} .
\end{aligned}
$$

Proof: The proof can be found in Appendix E.

To demonstrate that there is a one to one mapping between the set of feasible schedules $\mathcal{F}$ and the set of independent sets $\mathcal{I}$ of size $Z_{\text {tot }}$, we first show that each element of $\mathcal{F}$ is represented by a unique element in $\mathcal{I}$. We, then, show that each independent set can uniquely be represented by a feasible schedule.

Let the feasible schedule $\mathcal{S} \in \mathcal{F}$ be associated with the set of vertices $I$ in the conflict graph. Assume $\exists v \neq v^{\prime} \in I$ such that $v$ and $v^{\prime}$ are connected. From the connectivity conditions in the conflict graph, vertices $v$ and $v^{\prime}$ verify one of the following conditions

- CC1: $\delta\left(\varphi_{u}(v)-\varphi_{u}\left(v^{\prime}\right)\right)\left(1-\delta\left(\varphi_{c}(v)-\varphi_{c}\left(v^{\prime}\right)\right)\right)=1$ : this condition violates the constraint (A.3a) of the construction of $\mathcal{F}$.

- CC2: $\left(\varphi_{c}(v), \varphi_{b}(v), \varphi_{z}(v)\right)=\left(\varphi_{c}\left(v^{\prime}\right), \varphi_{b}\left(v^{\prime}\right), \varphi_{z}\left(v^{\prime}\right)\right)$ : this condition violates the constraint (A.3b) of the construction of $\mathcal{F}$.

- CC3: $\delta\left(\varphi_{u}(v)-\varphi_{u}\left(v^{\prime}\right)\right) \delta\left(\varphi_{z}(v)-\varphi_{z}\left(v^{\prime}\right)\right)=1$ : this condition violates the constraint (A.3c) of the construction of $\mathcal{F}$.

Therefore, each pair of vertices $v \neq v^{\prime} \in I$ are not connected which demonstrates that $I$ is an independent set of vertices in the conflict graph. Finally, from the construction constraint (A.3d), $\mathcal{S}$ and by extension $I$ have $Z_{\text {tot }}$ associations. Therefore, $I$ is a set of $Z_{\text {tot }}$ independent vertices which concludes that $I \in \mathcal{I}$. The uniqueness of $I$ follows directly from the bijection between the set of vertices in the graph and the set of associations in $\mathcal{A}$.

To establish the converse, let $I \in \mathcal{I}$ be an independent set of size $Z_{\text {tot }}$ and let $\mathcal{S}$ be its corresponding schedule. Using an argument similar to the one in previous paragraph, it can be easily shown that all the associations in $\mathcal{S}$ verify the constraints (A.3a), (A.3b), and (A.3c). Given that $I$ is of size $Z_{\mathrm{tot}}$, then $\mathcal{S}$ verify (A.3d) which concludes that $\mathcal{S} \in \mathcal{F}$. Uniqueness of the element is given by the same argument as earlier.

To conclude the proof, note that the weight of an independent set $I \in \mathcal{I}$ is equal to the objective function (A.1) and by extension to the original objective function (2). Therefore, the globally optimal solution of the joint scheduling problem in multi-cloud network (2) is equivalent to a maximum-weight independent set among the independent sets of size $Z_{\text {tot }}$ in the conflict graph.

\section{APPENDIX B PROOF OF THEOREM 2}

To show that the distributed solution reached by Algorithm 2 is the optimal solution to the scheduling problem (2), we first show that solving the maximum-weight independent set locally when users are assigned to a cloud like in the optimal solution to (2) will yield the optimal solution. Afterward, we show that Algorithm 2 assigns users to clouds as in the optimal solution. Combining the two above points concludes that the solution reached by Algorithm 2 is the optimal solution to (2). To finish the proof, we show that the running time of the algorithm is bounded.

First define $\mathcal{I}_{c}$ as the set of independent sets of size $B Z$ in the local conflict graph of cloud $c$. Let $I_{c} \in \mathcal{I}_{c}$ be an independent sets. The following lemma states the feasibility of the schedule $\mathcal{S}=\bigcup_{c \in \mathcal{C}} I_{c}$. 
Lemma 3. Let $I_{c} \in \mathcal{I}_{c}$ be an independent set of size $B Z$ in the local conflict graph of cloud $c \in \mathcal{C}$ (i.e., $\varphi_{c}(v)=c, \forall v \in I_{c}$ ) such that each user is assigned to at most a single cloud. In other words, for $c \neq c^{\prime}$, we have $\varphi_{u}(v) \neq \varphi_{u}\left(v^{\prime}\right), \forall v \in$ $I_{c}, v^{\prime} \in I_{c^{\prime}}$. The schedule $\mathcal{S}=\bigcup_{c \in \mathcal{C}} I_{c}$ is a feasible solution to the optimization problem (2).

Proof: The proof can be found in Appendix F.

Let $\mathcal{U}_{c}^{s}$ be the set of users scheduled in cloud $c$ at the optimal solution $X_{c u b z}^{*}$ of the optimization problem (2). The mathematical definition of this set is the following:

$$
\mathcal{U}_{c}^{s}=\left\{u \in \mathcal{U} \mid \exists(b, z) \in \mathcal{B} \times \mathcal{Z} \text { such that } X_{c u b z}^{*}=1\right\}
$$

Showing that solving the maximum-weight independent set locally when users are assigned to cloud like in the optimal solution to (2) yields the optimal solution is equivalent to showing the following. Assume that the set allowed users $\mathcal{U}_{c}^{s} \subseteq \mathcal{U}_{c}$ by cloud $c$ is set of users scheduled in that cloud at the optimal solution and let $I_{c} \in \mathcal{I}_{c}$ be the maximum-weight independent set of cloud $c$. We have to show that $\mathcal{S}=\bigcup_{c \in \mathcal{C}} I_{c}$ is the optimal scheduling. According to Theorem 1, the optimal solution of (2) can be written as follows:

$$
\max _{I \in \mathcal{I}} \sum_{v \in I} w(v)
$$

Let $\mathcal{U}_{s}=\bigcup_{c \in \mathcal{C}} \mathcal{U}_{c}^{s}$ be the set of all scheduled users in the optimal solution. We show that the optimal solution to (2) is the same if we consider $\mathcal{U}=\mathcal{U}_{s}$. Let $\mathcal{I}_{s}$ be the set of independent sets of size $C B Z$ in the conflict graph $\mathcal{G}\left(\mathcal{U}_{s}\right)$. Therefore, since $\mathcal{I}_{s} \subseteq \mathcal{I}$, the optimal solution can be written as:

$$
\max _{I \in \mathcal{I}_{s}} \sum_{v \in I} w(v) \leq \max _{I \in \mathcal{I}} \sum_{v \in I} w(v)
$$

However, for the solution $X_{\text {cubz }}^{*}$ we have $\max _{I \in \mathcal{I}_{s}} \sum_{v \in I} w(v)=\max _{I \in \mathcal{I}} \sum_{v \in I} w(v)$. Therefore, the optimal schedule when considering $\mathcal{U}=\mathcal{U}_{s}$ is the same as the optimal one of problem (2). We also have $\mathcal{I}_{s}=\bigcup_{c \in \mathcal{C}} \mathcal{I}_{c}$. Therefore, the optimal solution of (2) can be bounded by the following quantity:

$$
\begin{aligned}
\max _{I \in \mathcal{I}} \sum_{v \in I} w(v) & =\max _{I \in \mathcal{I}_{s}} \sum_{v \in I} w(v) \\
& =\max _{I \in \bigcup_{c \in \mathcal{C} \mathcal{I}_{c}}} \sum_{v \in I} w(v) \\
& \leq \sum_{c \in \mathcal{C}} \max _{I_{c} \in \mathcal{I}_{c}} \sum_{v \in I_{c}} w(v)
\end{aligned}
$$

From the feasibility of the optimal solution $X_{c u b z}^{*}$, we have $\mathcal{U}_{c}^{s} \cap \mathcal{U}_{c^{\prime}}^{s}=\varnothing, \forall c \neq c^{\prime}$. Therefore from Lemma 3, the schedule $\mathcal{S}$ is a feasible solution. In other words, the upper bound is achievable. Therefore, the schedule $\mathcal{S}=\bigcup_{c \in \mathcal{C}} I_{c}$ is the optimal solution to (2).

We now show that Algorithm 2 assigns users to clouds as in the optimal solution. Assume that a user $u$ scheduled in the cloud $c^{*}$ in the optimal solution is assigned to that cloud in Algorithm 2. In other words, we have $u \in \mathcal{U}_{c^{*}}^{s}$ and $u \notin \mathcal{U}_{c^{*}}$. This can happen only if at some time round $t$ in the algorithm, user $u$ is assigned to another cloud $c$. Hence, at some time round we have $u \in \mathcal{K}, c^{*}, c \in \hat{\mathcal{C}}(u)$ and the following equation holds:

$$
\begin{gathered}
\pi_{c u}+\sum_{\substack{c^{\prime} \in \hat{\mathcal{C}}(u) \\
c^{\prime} \neq c}} \bar{\pi}_{c^{\prime} u} \geq \pi_{c^{*} u}+\sum_{\begin{array}{c}
c^{\prime} \in \hat{\mathcal{C}}(u) \\
c^{\prime} \neq c^{*}
\end{array}} \bar{\pi}_{c^{\prime} u} \\
\pi_{c u}+\bar{\pi}_{c^{*} u}+\sum_{\substack{c^{\prime} \in \hat{\mathcal{C}}(u) \\
c^{\prime} \neq c, c^{*}}} \bar{\pi}_{c^{\prime} u} \geq \pi_{c^{*} u}+\bar{\pi}_{c u}+\sum_{\begin{array}{r}
c^{\prime} \in \hat{\mathcal{C}}(u) \\
c^{\prime} \neq c, c^{*}
\end{array}} \bar{\pi}_{c^{\prime} u} \\
\pi_{c u}+\bar{\pi}_{c^{*} u} \geq \pi_{c^{*} u}+\bar{\pi}_{c u}
\end{gathered}
$$

Let $\pi^{*}$, the objective function of the optimization problem (2) at the optimal solution $X_{c u b z}^{*}$, be decomposed as follows:

$$
\pi^{*}=\sum_{c^{\prime} \in \mathcal{C}} \pi_{c^{\prime}}^{*}=\sum_{\substack{c^{\prime} \in \mathcal{C} \\ c^{\prime} \neq c, c^{*}}}^{\pi^{*}} \pi_{c^{\prime}}^{*}+\pi_{c}^{*}+\pi_{c^{*}}^{*}
$$

Since user $u$ is scheduled to the cloud $c^{*}$ in the optimal solution then $\pi_{c^{*}}^{*}=\pi_{c^{*} u}^{*}$. Moreover, it is clear that $\pi_{c}^{*} \leq \bar{\pi}_{c u}$ since $\bar{\pi}_{c u}$ is the optimal schedule for cloud $c$ when it is not allowed to schedule user $u$. Therefore, the optimal objective function of the problem (2) is bounded by the following quantity:

$$
\pi^{*} \leq \sum_{\substack{c^{\prime} \in \mathcal{C} \\ c^{\prime} \neq c, c^{*}}} \pi_{c^{\prime}}^{*}+\bar{\pi}_{c u}+\pi_{c^{*} u}^{*}
$$

Moreover, it is clear that the merit $\pi_{c^{*} u}$ of user $u$ that is scheduled to cloud $c^{*}$, regardless of the feasibility of the whole schedule is higher than any other scheduling feasibility of the entire schedule. In particular, we have:

$$
\pi_{c^{*} u}^{*} \leq \pi_{c^{*} u}
$$

Substituting (B.8) in (B.7) then applying (B.5) yields the following inequality:

$$
\begin{gathered}
\pi^{*} \leq \sum_{\substack{c^{\prime} \in \mathcal{C} \\
c^{\prime} \neq c, c^{*}}} \pi_{c^{\prime}}^{*}+\bar{\pi}_{c u}+\pi_{c^{*} u} \\
\leq \sum_{\substack{c^{\prime} \in \mathcal{C} \\
c^{\prime} \neq c, c^{*}}} \pi_{c^{\prime}}^{*}+\pi_{c u}+\bar{\pi}_{c^{*} u}
\end{gathered}
$$

Now consider the scheduling in which user $u$ is scheduled to cloud $c$ and all the scheduling for clouds $c^{\prime} \neq c, c^{*}$ is the same. The merit function $\pi$ of such scheduling is:

$$
\pi=\sum_{\substack{c^{\prime} \in \mathcal{C} \\ c^{\prime} \neq c, c^{*}}} \pi_{c^{\prime}}^{*}+\pi_{c}+\pi_{c^{*}}
$$

Since user $u$ is scheduled to cloud $c$ then $\pi_{c}=\pi_{c u}$.

$$
\pi=\sum_{\substack{c^{\prime} \in \mathcal{C} \\ c^{\prime} \neq c, c^{*}}} \pi_{c^{\prime}}^{*}+\pi_{c u}+\pi_{c^{*}}
$$

The merit $\bar{\pi}_{c^{*} u}$ being the optimal benefit when user $c^{*}$ is not allowed to schedule user $u$ that it is greater than the merit of any schedule that do not schedule user $u$. In particular, since in $\pi_{c^{*}}$ user $u$ is not scheduled to cloud $c^{*}$ then we obtain:

$$
\bar{\pi}_{c^{*} u} \leq \pi_{c^{*}}
$$

Substituting (B.12) in (B.9), we obtain:

$$
\pi^{*} \leq \sum_{\substack{c^{\prime} \in \mathcal{C} \\ c^{\prime} \neq c, c^{*}}} \pi_{c^{\prime}}^{*}+\pi_{c u}+\pi_{c^{*}} \leq \pi,
$$

which is in contradiction with the fact that $p i^{*}$ is the optimal weight that that $\pi$ is the merit of a feasible schedule. Finally, we conclude that (B.5) do not hold, and that Algorithm 2 assigns users to clouds as in the optimal solution. 
To show that the optimal solution is reached by Algorithm 2 we combine the previous two results. First note that when the algorithm terminates we have $\mathcal{K}=\varnothing$. Let $I_{c}$ the maximumweight clique in each cloud. Using a proof similar to the one in (B.3), we can easily show that the maximum-weight clique $I_{c}$ do not change if we consider the set $\tilde{\mathcal{U}}_{c}$ of users used in the scheduling $I_{c}$ instead of $\mathcal{U}_{c}$. Since $\mathcal{K}=\varnothing$ then $\tilde{\mathcal{U}}_{c} \cap \tilde{\mathcal{U}}_{c^{\prime}}=\varnothing$. Moreover, we show above that $\mathcal{U}_{c}^{s} \subseteq \tilde{\mathcal{U}}_{c}$. As shown earlier, this condition is equivalent to solving optimally the scheduling problem. Finally, the optimal solution can be reached by Algorithm 2.

To show that the running time of the algorithm is bounded it this sufficient to note that at each time round of the algorithm, since $\mathcal{K} \neq \varnothing$, then $\exists c, u$ such that $\mathcal{U}_{c}=\mathcal{U}_{c} \backslash\{u\}$. In other words, $\left|\mathcal{U}_{c}\right|=\left|\mathcal{U}_{c}\right|-1$ Since that $\left|\mathcal{U}_{c}\right|$ is lower bounded by $\left|\mathcal{U}_{c}^{s}\right|$. Therefore, the running time of Algorithm 2 is bounded by $C\left(\max _{c}\left|\mathcal{U}_{c}\right|-\min _{c}\left|\mathcal{U}_{c}^{s}\right|\right)$. Clearly, we have $\max _{c}\left|\mathcal{U}_{c}\right|=U$. We now show that $\min _{c}\left|\mathcal{U}_{c}^{s}\right|=Z$.

We show that for a schedule $\mathcal{S}$ to be feasible, a user $u$ assigned to cloud $c$ can be scheduled to at most $Z$ PZs across the different BSs in $c$. Assume that user $u$ is connected to $Z^{\prime}>Z$ PZs then the schedule $\mathcal{S}$ contains $Z^{\prime}$ vertices $v$ such that $\varphi_{u}(v)=u$. The number of PZ index being $Z$ then from the pigeon-hole principle $\exists v, v^{\prime}$ such that $\varphi_{z}(v)=\varphi_{z}\left(v^{\prime}\right)$. From the graph connectivity condition $\mathrm{C} 3$, we have $\delta\left(\varphi_{u}(v)-\right.$ $\left.\varphi_{u}\left(v^{\prime}\right)\right) \delta\left(\varphi_{z}(v)-\varphi_{z}\left(v^{\prime}\right)\right)=1$. Therefore, vertices $v$ and $v^{\prime}$ are connected which is in contradiction with the fact that the schedule $\mathcal{S}$ is a feasible solution and hence an independent set. Finally, The running time of the algorithm is bounded by $C(U-B)$. Note that since $U \geq B$ for the problem to have at least one solution, then the quantity is always positive.

\section{APPENDIX C \\ PROOF OF COROLlaRY 1}

To prove this corollary, it is sufficient to show that Algorithm 3 converges. Afterwards, applying the result of Lemma 3 guarantee the feasibility of the solution. At time round of the algorithm such that $\mathcal{K} \neq \varnothing$, we have $\exists c^{*}, u$ such that $\forall c \neq c^{*}$ we have $\mathcal{U}_{c}=\mathcal{U}_{c} \backslash\{u\}$. Therefore, the running time of the algorithm is bounded by $\max _{c}\left|\mathcal{U}_{c}\right|$ which is equal to $U$ from Theorem 2. Therefore, Algorithm 3 converges and outputs the independent sets $I_{c} \in \mathcal{I}_{c}$. From Lemma 3, the solution $\mathcal{S}=\bigcup_{c \in \mathcal{C}} I_{c}$ is a feasible solution to the optimization problem (2) since $\mathcal{U}_{c} \cap \mathcal{U}_{c^{\prime}}=\varnothing, \forall c \neq c^{\prime}$.

\section{APPENDIX D \\ PROOF OF LEMMA 1}

Note that the constraints (8b), (8c) and (8d) of the optimization problem (8) are the same constraints as (2d), (2e) and (2f), respectively, in the original optimization problem (2). Therefore, this lemma can be proved using steps similar to the one used in Theorem 1.

Let $\mathcal{F} \subset \mathcal{P}(\mathcal{A})$ be the set of feasible schedules. Given the mapping between the original constraints of the problem and the constraints of constructing the set $\mathcal{F}$ illustrated in Lemma 2, it can be easily shown that problem (8) can be written as follows:

$$
\begin{aligned}
& \max _{\mathcal{S} \in \mathcal{P}(\mathcal{A})} \sum_{a \in \mathcal{S}} \pi(a) \\
& \text { s.t. } \mathcal{S} \in \mathcal{F},
\end{aligned}
$$

where $\mathcal{F}$ is the set of feasible schedules defined as follows:

$$
\begin{aligned}
& \mathcal{F}=\left\{\mathcal{S} \in \mathcal{P}(\mathcal{A}) \text { such that } \forall a \neq a^{\prime} \in \mathcal{S}\right. \\
& \left(\varphi_{c}(a), \varphi_{b}(a), \varphi_{z}(a)\right) \neq\left(\varphi_{c}\left(a^{\prime}\right), \varphi_{b}\left(a^{\prime}\right), \varphi_{z}\left(a^{\prime}\right)\right), \\
& \delta\left(\varphi_{u}(a)-\varphi_{u}\left(a^{\prime}\right)\right) \delta\left(\varphi_{z}(a)-\varphi_{z}\left(a^{\prime}\right)\right)=0 \\
& \left.|\mathcal{S}|=Z_{\text {tot }}\right\} .
\end{aligned}
$$

Let the reduced conflict graph be constructed by generating a vertex of each association $a \in \mathcal{A}$ and connecting two distinct vertices $v$ and $v^{\prime}$ if one of the following two conditions holds:

- CC2: $\left(\varphi_{c}(v), \varphi_{b}(v), \varphi_{z}(v)\right)=\left(\varphi_{c}\left(v^{\prime}\right), \varphi_{b}\left(v^{\prime}\right), \varphi_{z}\left(v^{\prime}\right)\right)$.

- CC3: $\delta\left(\varphi_{u}(v)-\varphi_{u}\left(v^{\prime}\right)\right) \delta\left(\varphi_{z}(v)-\varphi_{z}\left(v^{\prime}\right)\right)=1$.

Define $\mathcal{I}$ as the set of the independent set of vertices of size $Z_{\text {tot }}$ in the reduced conflict graph. Following steps similar to the one used in Theorem 1, it can be shown that there is a one to one mapping between the set of feasible schedule $\mathcal{F}$ and the set $\mathcal{I}$ and that the objective function is represented by the sum of the weight of the vertices in the independent set. As a conclusion, the optimal solution to the scheduling problem (8) in signal-level coordinated cloud-enabled network is the maximum-weight independent set of size $C B Z$ in the reduced conflict graph.

\section{APPENDIX E \\ PROOF OF LEMMA 2}

To prove this lemma, it is sufficient to prove to that the objective function and the constraints of (2) are equivalent to those of the optimization problem (A.1). The objective function of (2) is equivalent to the one of (A.1) as shown in the following equation:

$$
\sum_{c, u, b, z} \pi_{c u b z} X_{c u b z}=\sum_{a \in \mathcal{A}} \pi(a) X(a)=\sum_{a \in \mathcal{S}} \pi(a),
$$

where $X(a)$ is defined in the same manner as $\pi(a)$, i.e., $X(a)=X_{c u b z}$ for $a=(c, u, b, z) \in \mathcal{A}$ and $\mathcal{S}=\{a \in$ $\mathcal{A} \mid X(a)=1\}$. Therefore, the two objective functions are equivalent:

$$
\max \sum_{c, u, b, z} \pi_{c u b z} X_{c u b z}=\max _{\mathcal{S} \in \mathcal{P}(\mathcal{A})} \sum_{a \in \mathcal{S}} \pi(a) .
$$

In what follows, the constraints (2b) and (2c) are shown to be equivalent to the constraint (A.3a), the constraint (2d) is proven to be equivalent to (A.3b) and (A.3d). Finally to conclude the proof, (2e) is demonstrated to be the same constraint as (A.3c).

Define $\mathcal{S}_{c u} \subset \mathcal{S}$ as the set of associations in schedule $\mathcal{S}$ concerning the $c$ th cloud and the $u$ th user. The expression of the set is the following:

$$
\mathcal{S}_{c u}=\left\{a \in \mathcal{S} \mid \varphi_{c}(a)=c, \varphi_{u}(a)=u\right\} .
$$

Let $\mathcal{S}_{u} \subset \mathcal{P}(\mathcal{S})$ be the set of all the set concerning user $u$ defined as:

$$
\mathcal{S}_{u}=\left\{\mathcal{S}_{c u}, c \in \mathcal{C}\right\} .
$$

The constraints (2b) (i.e., $Z_{c u}=1-\delta\left(\sum_{b, z} X_{c u b z}\right)$ ) and (2c) 
(i.e., $\sum_{c} Z_{c u} \leq 1$ ) are equivalent to the following constraint

$$
Z_{c u}=1-\delta\left(\sum_{b, z} X_{c u b z}\right) \leq 1 \Leftrightarrow\left|\mathcal{S}_{u}\right| \leq 1, \forall u .
$$

We now show that the inequality $\left|\mathcal{S}_{u}\right| \leq 1$ is equivalent to the following equality $\forall a \neq a^{\prime} \in \mathcal{S}$ :

$$
\delta\left(\varphi_{u}(a)-\varphi_{u}\left(a^{\prime}\right)\right)\left(1-\left(\delta\left(\varphi_{c}(a)-\varphi_{c}\left(a^{\prime}\right)\right)\right)=0\right.
$$

First note that if $a \in \mathcal{S}_{u}$ and $a^{\prime} \in \mathcal{S}_{u^{\prime}}$ with $u \neq u^{\prime}$, then $\varphi_{u}(a) \neq \varphi_{u}\left(a^{\prime}\right)$ which concludes that (E.6) holds for such $a$ and $a^{\prime}$. Now let $a \neq a^{\prime} \in \mathcal{S}_{u}$. Since $\left|\mathcal{S}_{u}\right| \leq 1$ then $\exists$ unique $c \in \mathcal{C}$ such that $\mathcal{S}_{c u} \neq \varnothing$. Hence $a \neq a^{\prime} \in \mathcal{S}_{c u}$, i.e., $\varphi_{c}(a)=\varphi_{c}\left(a^{\prime}\right)$ which concludes that (E.6) holds for such $a$ and $a^{\prime}$. Given that $\mathcal{S}$ can be written as $\bigcup_{u} \mathcal{S}_{u}$, then (E.6) is valid $\forall a \neq a^{\prime} \in \mathcal{S}$. Combining (E.5) and (E.6) proves that the constraints $(2 \mathrm{~b})$ and $(2 \mathrm{c})$ are equivalent to the constraint (A.3a).

Define $\mathcal{S}_{c b z} \subset \mathcal{S}$ as the set of associations in schedule $\mathcal{S}$ concerning the $z$ th $\mathrm{PZ}$ in the $b$ th BS connected to the $c$ th cloud. The expression of the set is the following:

$$
\mathcal{S}_{c b z}=\left\{a \in \mathcal{S} \mid \varphi_{c}(a)=c, \varphi_{b}(a)=b, \varphi_{z}(a)=z\right\} \text {. }
$$

The constraint (2d) can be written as a function of the partial schedules as follows:

$$
\sum_{u} X_{c u b z}=1 \Leftrightarrow\left|\mathcal{S}_{c b z}\right|=1, \forall(c, b, z) .
$$

Assume $\exists a \neq a^{\prime} \in \mathcal{S}$ such that $\varphi_{c}(a)=\varphi_{c}\left(a^{\prime}\right), \varphi_{b}(a)=$ $\varphi_{b}\left(a^{\prime}\right)$, and $\varphi_{z}(a)=\varphi_{z}\left(a^{\prime}\right)$. It is clear that $a, a^{\prime} \in \mathcal{S}_{c b z}$ where $c=\varphi_{c}(a), b=\varphi_{b}(a)$, and $z=\varphi_{z}(a)$. However, from (E.8), we have $\left|\mathcal{S}_{c b z}\right|=1$. Therefore, $a=a^{\prime}$ which concludes that, $\forall a \neq a^{\prime} \in \mathcal{S}$, we have:

$$
\left(\varphi_{c}(a), \varphi_{b}(a), \varphi_{z}(a)\right) \neq\left(\varphi_{c}\left(a^{\prime}\right), \varphi_{b}\left(a^{\prime}\right), \varphi_{z}\left(a^{\prime}\right)\right) .
$$

We now show that $\mathcal{S}_{c b z} \cap \mathcal{S}_{c^{\prime} b^{\prime} z^{\prime}}=\varnothing$ for all sets in which at least one of the following holds: $c \neq c^{\prime}$, and/or $b \neq b^{\prime}$, and/or $z \neq z^{\prime}$. From (E.8), both sets contain a single association, hence $\mathcal{S}_{c b z} \cap \mathcal{S}_{c^{\prime} b^{\prime} z^{\prime}} \neq \varnothing$ means that $\mathcal{S}_{c b z}=\mathcal{S}_{c^{\prime} b^{\prime} z^{\prime}}$ which do not hold since $c \neq c^{\prime}$, and/or $b \neq b^{\prime}$, and/or $z \neq z^{\prime}$. As a conclusion, the cardinality of the schedule $\mathcal{S}$ can be written as:

$$
|\mathcal{S}|=\left|\bigcup_{c, b, z} \mathcal{S}_{c b z}\right|=\bigcup_{c, b, z}\left|\mathcal{S}_{c b z}\right|=C B Z=Z_{\text {tot }} .
$$

The combination of equations (E.8), (E.9) and (E.10) shows that the constraint (2d) is equivalent to (A.3b) and (A.3d).

Define $\mathcal{S}_{u z} \subset \mathcal{S}$ as the set of associations in schedule $\mathcal{S}$ concerning the $u$ th user scheduled in the $z$ th PZ of one of the connected BS. The expression of the set is the following:

$$
\mathcal{S}_{u z}=\left\{a \in \mathcal{S} \mid \varphi_{u}(a)=u, \varphi_{z}(a)=z\right\} .
$$

The constraint (2e) can be written as a function of the partial schedules as follows:

$$
Y_{u z}=\sum_{c b} X_{c u b z} \leq 1 \Leftrightarrow\left|\mathcal{S}_{u z}\right| \leq 1, \forall(u, z) .
$$

To conclude the proof, it is sufficient to show that, if $\left|\mathcal{S}_{u z}\right| \leq$ 1, $\forall(u, z)$, then the following equation holds for $a \neq a^{\prime} \in \mathcal{S}$ :

$$
\delta\left(\varphi_{u}(a)-\varphi_{u}\left(a^{\prime}\right)\right) \delta\left(\varphi_{z}(a)-\varphi_{z}\left(a^{\prime}\right)\right)=0 .
$$

Let the schedule be partitioned into partial schedules as follows $\mathcal{S}=\bigcup_{u z} \mathcal{S}_{u z}$. For $a \in \mathcal{S}_{u z}$ and $a^{\prime} \in \mathcal{S}_{u^{\prime} z^{\prime}} \neq \mathcal{S}_{u z}$, it is clear that either $u \neq u^{\prime}$ and/or $z \neq z^{\prime}$. Hence, equality (E.13) holds for all $a \in \mathcal{S}_{u z}$ and $a^{\prime} \in \mathcal{S}_{u^{\prime} z^{\prime}} \neq \mathcal{S}_{u z}$. Given that
$\left|\mathcal{S}_{u z}\right| \leq 1$, then $\nexists a \neq a^{\prime} \in \mathcal{S}_{u z}, \forall(u, z)$ which concludes that (E.13) is verified. The combination of equations (E.12), and (E.13) shows that the constraint (2e) is equivalent to (A.3c).

\section{APPENDIX F \\ PROOF OF LEMMA 3}

To show this lemma, according to Theorem 1, we only need to show that the schedule $\mathcal{S}=\bigcup_{c \in \mathcal{C}} I_{c}$ is an independent set of size $C B Z$ in the conflict graph. Since $I_{c}, \forall c \in \mathcal{C}$ is an independent set in the local conflict graph then proving that $\mathcal{S}$ is an independent set in the conflict graph boils down to proving that there are no connections between any pair of vertices belonging to different local independent set $I_{c}$ and $I_{c^{\prime}}, c \neq c^{\prime}$.

Let $\mathcal{G}_{c}$ and $\mathcal{G}_{c^{\prime}}$ be two distinct local conflict graphs (i.e., $\left.c \neq c^{\prime}\right)$. We show that if $v \in \mathcal{G}_{c}$ and $v^{\prime} \in \mathcal{G}_{c^{\prime}}$ are connected then $\varphi_{u}(v)=\varphi_{u}\left(v^{\prime}\right)$. From the connectivity conditions of vertices, $v$ and $v^{\prime}$ are connected if and only if at least one of the following conditions is verified:

- $\mathrm{C} 1: \delta\left(\varphi_{u}(v)-\varphi_{u}\left(v^{\prime}\right)\right)\left(1-\delta\left(\varphi_{c}(v)-\varphi_{c}\left(v^{\prime}\right)\right)\right)=1$.

- C2: $\left(\varphi_{c}(v), \varphi_{b}(v), \varphi_{z}(v)\right)=\left(\varphi_{c}\left(v^{\prime}\right), \varphi_{b}\left(v^{\prime}\right), \varphi_{z}\left(v^{\prime}\right)\right)$.

- C3: $\delta\left(\varphi_{u}(v)-\varphi_{u}\left(v^{\prime}\right)\right) \delta\left(\varphi_{z}(v)-\varphi_{z}\left(v^{\prime}\right)\right)=1$.

Clearly condition C2 cannot be satisfied since $\varphi_{c}(v)=$ $c \neq c^{\prime}=\varphi_{c}\left(v^{\prime}\right)$. Now assume that $\varphi_{u}(v) \neq \varphi_{u}\left(v^{\prime}\right)$, then $\delta\left(\varphi_{u}(v)-\varphi_{u}\left(v^{\prime}\right)\right)=0$. This last equality concludes that conditions $\mathrm{C} 1$ and $\mathrm{C} 3$ are not satisfied and hence the vertices not connected, which is a contradiction with the initial assumption. Therefore, $\varphi_{u}(v)=\varphi_{u}\left(v^{\prime}\right)$ for vertices $v$ and $v^{\prime}$ belonging to different local conflict graphs $\mathcal{G}_{c}$ and $\mathcal{G}_{c^{\prime}}$.

Given that in the schedule $\mathcal{S}$, we have $\varphi_{u}(v) \neq$ $\varphi_{u}\left(v^{\prime}\right), \forall v \in I_{c}, v^{\prime} \in I_{c^{\prime}}$ then there are no connections between any pair of vertices belonging to different local independent set $I_{c}$ and $I_{c^{\prime}}$. Therefore, $\mathcal{S}$ is an independent set in the conflict graph which size is equal to the sum of size of the local independent sets $I_{c}$. In other words, $\mathcal{S}$ is an independent set of size $Z_{\text {tot }}=C B Z$ which concludes that it is a feasible solution to the optimization problem (2).

\section{REFERENCES}

[1] A. Douik, H. Dahrouj, T. Y. Al-Naffouri, and M.-S. Alouini, "Multicloud coordinating via joint scheduling for the downlink of radio-access networks," IEEE Global Telecommunications Conference (GLOBECOM' 2015), San Diego, CA, USA,, 2015.

[2] J. Andrews, S. Buzzi, W. Choi, S. Hanly, A. Lozano, A. Soong, and J. Zhang, "What will 5G be?" IEEE Journal on Selected Areas in Communications, vol. 32, no. 6, pp. 1065-1082, June 2014.

[3] F. Boccardi, R. Heath, A. Lozano, T. Marzetta, and P. Popovski, "Five disruptive technology directions for 5G," IEEE Communications Magazine, vol. 52, no. 2, pp. 74-80, February 2014.

[4] M. Peng, K. Zhang, J. Jiang, J. Wang, and W. Wang, "Energy-efficient resource assignment and power allocation in heterogeneous cloud radio access networks," IEEE Transactions on Vehicular Technology, vol. PP, no. 99, pp. 1-1, 2014.

[5] D. Gesbert, S. Hanly, H. Huang, S. Shamai Shitz, O. Simeone, and W. Yu, "Multi-cell mimo cooperative networks: A new look at interference," IEEE Journal on Selected Areas in Communications, vol. 28, no. 9, pp. 1380-1408, December 2010.

[6] H. Dahrouj, A. Douik, O. Dhifallah, T. Y. Al-Naffouri, and M.-S. Alouini, "Resource allocation in heterogeneous cloud radio access networks: advances and challenges," IEEE Wireless Communications, vol. 22, no. 3, pp. 66-73, June 2015. 
[7] S.-H. Park, O. Simeone, O. Sahin, and S. Shamai, "Inter-cluster design of precoding and fronthaul compression for cloud radio access networks," IEEE Wireless Communications Letters, vol. 3, no. 4, pp. 369-372, Aug 2014.

[8] — - "Joint precoding and multivariate backhaul compression for the downlink of cloud radio access networks," IEEE Transactions on Signal Processing, vol. 61, no. 22, pp. 5646-5658, Nov 2013.

[9] Y. Shi, J. Zhang, and K. Letaief, "Group sparse beamforming for green cloud-ran," IEEE Transactions on Wireless Communications, vol. 13, no. 5, pp. 2809-2823, May 2014.

[10] W. Yu, T. Kwon, and C. Shin, "Multicell coordination via joint scheduling, beamforming, and power spectrum adaptation," IEEE Transactions on Wireless Communications, vol. 12, no. 7, pp. 1-14, July 2013.

[11] H. Dahrouj, W. Yu, T. Tang, J. Chow, and R. Selea, "Coordinated scheduling for wireless backhaul networks with soft frequency reuse," in Proc. of the 21st Europea Signal Processing Conference (EUSIPCO 2013), Marrakech, Morocco, Sept 2013, pp. 1-5.

[12] A. Douik, H. Dahrouj, T. Y. Al-Naffouri, and M.-S. Alouini, "Coordinated scheduling for the downlink of cloud radio-access networks," Proc. of IEEE International Conference on Communications (ICC' 2015), London, UK., 2015.

[13] B. Rengarajan, A. Stolyar, and H. Viswanathan, "Self-organizing dynamic fractional frequency reuse on the uplink of ofdma systems," in Proc. of 2010 44th Annual Conference on Information Sciences and Systems (CISS' 2010), Princeton, New Jersey, USA, March 2010, pp. $1-6$.

[14] D. P. Bertsekas, "The auction algorithm: A distributed relaxation method for the assignment problem," Annals of Operations Research, vol. 14, pp. 105-123, 1988.

[15] O. Dhifallah, H. Dahrouj, T. Y. Al-Naffouri, and M.-S. Alouini, "Decentralized group sparse beamforming for multi-cloud radio access networks," in Proc. of IEEE Globecom, San Diego, USA, Dec 2015.

[16] J. Zhang, R. Chen, J. G. Andrews, A. Ghosh, and R. W. Heath, "Networked MIMO with clustered linear precoding," IEEE Transactions on Wireless Communications, vol. 8, no. 4, pp. 1910-1921, April 2009.

[17] W. Choi and J. Andrews, "The capacity gain from intercell scheduling in multi-antenna systems," IEEE Transactions on Wireless Communications, vol. 7, no. 2, pp. 714-725, February 2008.

[18] B. Dai and W. Yu, "Sparse beamforming for limited-backhaul network mimo system via reweighted power minimization," in Proc. of IEEE Global Telecommunications Conference (GLOBECOM' 2013), Atlanta, GA, USA, Dec 2013, pp. 1962-1967.

[19] S. Kiani and D. Gesbert, "Optimal and distributed scheduling for multicell capacity maximization," IEEE Transactions on Wireless Communications, vol. 7, no. 1, pp. 288-297, Jan 2008.

[20] R. Bendlin, Y.-F. Huang, M. Ivrlac, and J. Nossek, "Fast distributed multi-cell scheduling with delayed limited-capacity backhaul links," in Proc. of IEEE International Conference on Communications (ICC' 2009), Dresden, Germany, June 2009, pp. 1-5.

[21] J. Papandriopoulos and J. Evans, "Scale: A low-complexity distributed protocol for spectrum balancing in multiuser dsl networks," IEEE Transactions on Information Theory, vol. 55, no. 8, pp. 3711-3724, Aug 2009.

[22] J. Mundarath, P. Ramanathan, and B. Van Veen, "A distributed downlink scheduling method for multi-user communication with zero-forcing beamforming," IEEE Transactions on Wireless Communications, vol. 7, no. 11, pp. 4508-4521, November 2008.

[23] Y. Xu, J. Wang, Q. Wu, A. Anpalagan, and Y. D. Yao, "Opportunistic spectrum access in cognitive radio networks: Global optimization using local interaction games," IEEE Journal of Selected Topics in Signal Processing, vol. 6, no. 2, pp. 180-194, April 2012.

[24] Y. Xu, A. Anpalagan, Q. Wu, L. Shen, Z. Gao, and J. Wang, "Decisiontheoretic distributed channel selection for opportunistic spectrum access: Strategies, challenges and solutions," IEEE Communications Surveys Tutorials, vol. 15, no. 4, pp. 1689-1713, Fourth 2013.

[25] D. Gesbert, S. Kiani, A. Gjendemsjo, and G. ien, "Adaptation, coordination, and distributed resource allocation in interference-limited wireless networks," Proceedings of the IEEE, vol. 95, no. 12, pp. 2393-2409, Dec 2007.

[26] J. Huang, R. Berry, and M. Honig, "Distributed interference compensation for wireless networks," IEEE Journal on Selected Areas in Communications, vol. 24, no. 5, pp. 1074-1084, May 2006.

[27] C. Shi, R. Berry, and M. Honig, "Distributed interference pricing for ofdm wireless networks with non-separable utilities," in Proc. of 42nd Annual Conference on Information Sciences and Systems (CISS' 2008), Princeton, New Jersey, USA, March 2008, pp. 755-760.
[28] J. Yuan and W. Yu, "Distributed cross-layer optimization of wireless sensor networks: A game theoretic approach," in Proc. of IEEE Global Telecommunications Conference (GLOBECOM'2006), San Francisco, California, USA, Nov 2006, pp. 1-5.

[29] F. V. Fomin, F. Grandoni, and D. Kratsch, "A measure \& conquer approach for the analysis of exact algorithms," Journal of the ACM, vol. 56, no. 5, pp. 25:1-25:32, Aug. 2009.

[30] N. Bourgeois, B. Escoffier, V. T. Paschos, and J. M. M. van Rooij, "A bottom-up method and fast algorithms for max independent set," in Proc. of the 12th Scandinavian Conference on Algorithm Theory (SWAT" 2010), Bergen, Norway.

[31] P. jun Wan, X. Jia, G. Dai, H. Du, and O. Frieder, "Fast and simple approximation algorithms for maximum weighted independent set of links," in Proc. of 33th IEEE Conference on Computer Communications (INFOCOM' 2009), Toronto, canada, April 2014, pp. 1653-1661.

[32] N. Esfahani, P. Mazrooei, K. Mahdaviani, and B. Omoomi, "A note on the p-time algorithms for solving the maximum independent set problem," in Proc. of 2nd Conference on Data Mining and Optimization (DMO' 2009), Bandar Baru Bangi, Malaysia, Oct 2009, pp. 65-70.

[33] A. Douik, H. Dahrouj, T. Y. Al-Naffouri, and M. S. Alouini, "Coordinated scheduling and power control in cloud-radio access networks," IEEE Transactions on Wireless Communications, vol. 15, no. 4, pp. 2523-2536, April 2016

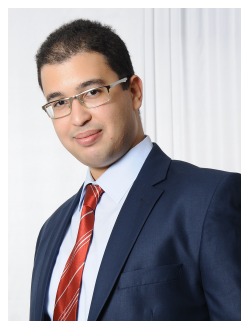

Ahmed Douik (S'13) received the Eng. degree in electronic and communication engineering (with first class honors) from the Ecole Polytechnique de Tunisie, Tunisia, in 2013, the M.S. degree in electrical engineering from King Abdullah University of Science and Technology, Thuwal, Saudi Arabia, in 2015. He is now pursuing his Ph.D. at the California Institute of Technology, Pasadena, CA, USA. His research interests include cloud-radio access networks, network coding, single and multi-hop transmissions, and cooperation communication.

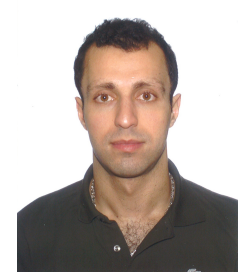

Hayssam Dahrouj (S'02, M'11, SM'15) received his B.E. degree (with high distinction) in computer and communications engineering from the American University of Beirut (AUB), Lebanon, in 2005, and his $\mathrm{Ph} . \mathrm{D}$. degree in electrical and computer engineering from the University of Toronto (UofT), Canada, in 2010. In May 2015, he joined the Department of Electrical and Computer Engineering at Effat University as an assistant professor, and also became a visiting scholar at King Abdullah University of Science and Technology (KAUST). Between April 2014 and May 2015, he was with the Computer, Electrical and Mathematical Sciences and Engineering group at KAUST as a research associate. Prior to joining KAUST, he was an industrial postdoctoral fellow at UofT, in collaboration with BLiNQ Networks Inc., Kanata, Canada, where he worked on developing practical solutions for the design of non-line-of sight wireless backhaul networks. His contributions to the field led to five patents. During his doctoral studies at UofT, he pioneered the idea of coordinated beamforming as a means of minimizing intercell interference across multiple base stations. The journal paper on this subject was ranked second in the 2013 IEEE Marconi paper awards in wireless communications. Dr. Dahrouj is the recipient of both the faculty award of excellence in research and the faculty award of excellence in teaching at Effat University, May 2017. His main research interests include cloud radio access networks, backhaul systems, cross-layer optimization, cooperative networks, convex optimization, distributed algorithms, and freespace optical communications. 


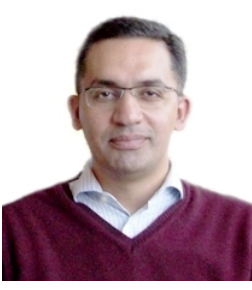

Tareq Y. Al-Naffouri (M'10) Tareq Al-Naffouri received the B.S. degrees in mathematics and electrical engineering (with first honors) from King Fahd University of Petroleum and Minerals, Dhahran, Saudi Arabia, the M.S. degree in electrical engineering from the Georgia Institute of Technology, Atlanta, in 1998, and the Ph.D. degree in electrical engineering from Stanford University, Stanford, CA, in 2004.

He was a visiting scholar at California Institute of Technology, Pasadena, CA, from January to August 2005 and during summer 2006. He was a Fulbright Scholar at the University of Southern California from February to September 2008. He has held internship positions at NEC Research Labs, Tokyo, Japan, in 1998, Adaptive Systems Lab, University of California at Los Angeles in 1999, National Semiconductor, Santa Clara, CA, in 2001 and 2002, and Beceem Communications Santa Clara, CA, in 2004. He is currently an Associate professor at the Electrical Engineering Department, King Abdullah University of Science and Technology (KAUST). His research interests lie in the areas of sparse, adaptive, and statistical signal processing and their applications and in network information theory. He has over 150 publications in journal and conference proceedings, 9 standard contributions, 10 issued patents, and 6 pending.

Dr. Al-Naffouri is the recipient of the IEEE Education Society Chapter Achievement Award in 2008 and Al-Marai Award for innovative research in communication in 2009. Dr. Al-Naffouri has also been serving as an Associate Editor of Transactions on Signal Processing since August 2013.

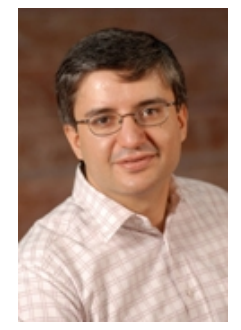

Mohamed-Slim Alouini (S'94-M'98-SM'03-F'09) was born in Tunis, Tunisia. He received the Ph.D. degree in electrical engineering from the California Institute of Technology (Caltech), Pasadena, CA, USA, in 1998. He served as a faculty member at the University of Minnesota, Minneapolis, MN, USA, then in the Texas A\&M University at Qatar, Education City, Doha, Qatar, before joining King Abdullah University of Science and Technology (KAUST), Thuwal, Makkah Province, Saudi Arabia, as a Professor of electrical engineering in 2009. His current research interests include the modeling, design, and performance analysis of wireless communication systems. 